\title{
Superconductivity and a Mott Transition in a Hubbard Model on an Anisotropic Triangular Lattice
}

\author{
Tsutomu Watanabe ${ }^{1,2 *}$, Hisatoshi Yokoyama ${ }^{3}$, Yukio Tanaka ${ }^{1,2}$ and Jun-ichiro Inoue ${ }^{1}$ \\ ${ }^{1}$ Department of Applied Physics, Nagoya University, Nagoya 464-8603 \\ ${ }^{2}$ CREST Japan Science and Technology Corporation (JST) \\ ${ }^{3}$ Department of Physics, Tohoku University, Sendai 980-8578
}

\begin{abstract}
A half-filled-band Hubbard model on an anisotropic triangular lattice ( $t$ in two bond directions and $t^{\prime}$ in the other) is studied using an optimization variational Monte Carlo method, to consider a Mott transition and superconductivity arising in $\kappa$-(BEDT-TTF $)_{2} \mathrm{X}$. Adopting wave functions with doublon-holon binding factors, we reveal that a first-order Mott (conductorto-nonmagnetic insulator) transition takes place at $U=U_{\mathrm{c}}$, approximately of the bandwidth, for a wide $t^{\prime} / t$ range. This transition is not directly connected to magnetism. Robust $d$-wave superconductivity appears in a restricted parameter range: immediately below $U_{\mathrm{c}}$ and the moderate strength of frustration $\left(0.4 \lesssim t^{\prime} / t \lesssim 0.7\right)$, where short-range antiferromagnetic correlation sufficiently develops but does not come to a long-range order. The relevance to experiments is also discussed.
\end{abstract}

KEYWORDS: Hubbard model, anisotropic triangular lattice, variational Monte Carlo method, superconductivity, $d_{x^{2}-y^{2}}$ wave, antiferromagnetic correlation, Mott transition, $\kappa$-(BEDT-TTF $)_{2} \mathrm{X}$

\section{Introduction}

Organic layered compounds made of BEDT-TTF (ET) molecules present various characteristics of correlated electron systems. Among them, $\kappa$-type compounds, ${ }^{1-3}$ $\kappa$ - $(\mathrm{ET})_{2} \mathrm{X}(\mathrm{X}$ denotes an anion), have intriguing properties as follows: (1) They have good two-dimensionality in conductivity with a frustrated lattice structure. (2) Most

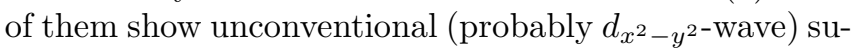
perconductivity (SC) as high as $10 \mathrm{~K}$, with pseudo-gap behavior for $T>T_{\mathrm{c}}$. These two points are closely akin to high- $T_{\mathrm{c}}$ cuprates. (3) A series of $\kappa$-(ET) salts give rise to superconductor-to-antiferromagnetic (AF) insulator transitions, through the chemical substitution of $\mathrm{X}$, or applied pressure. (4) It was recently found that a compound $\left[\mathrm{X}=\mathrm{Cu}_{2}(\mathrm{CN})_{3}\right]$ preserves an insulating state without magnetic order down to a quite low temperature $(23 \mathrm{mK}) .^{4}$

About a decade ago, Kino and Fukuyama ${ }^{5}$ studied the electronic structure of ET salts, applying a HartreeFock approximation to four-band Hubbard models, and found that if the dimerization between ET molecules is sufficiently strong, the conducting plane of $\kappa$-type compounds is described well by a single-band Hubbard model on an anisotropic triangular lattice (the hopping integral is $t$ in two bond directions and $t^{\prime}$ in the other) with the electron density of half filling. The Hartree-Fock approximation can describe a metal-to-AF insulator transition. Afterward, this model has been studied with various theories. Fluctuation exchange approximation (FLEX $)^{6,7}$ and a quantum Monte Carlo method $^{8}$ have yielded a re-

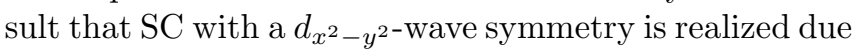
to AF spin correlation (or fluctuation), as in the case of cuprates. Actually, experimental studies using NMR, ${ }^{9-11}$ thermal conductivity, ${ }^{12}$ and tunneling spectroscopy measurements ${ }^{13-15}$ have found unconventional SC with nodes of the gap in $\kappa$-(ET) $)_{2} \mathrm{X}$.

However, it is difficult to deal with essential aspects of a strong correlation, particularly Mott transitions ${ }^{16}$ by Hartree-Fock or FLEX approximation. In contrast, dynamical cluster approximation (DCA $)^{17}$ and path integral renormalization group calculation (PIRG) ${ }^{18}$ have shown first-order metal-to-nonmagnetic-insulator transitions for intermediately and highly frustrated cases. However, both DCA and PIRG have not reached a definite conclusion regarding $\mathrm{SC}$ in the vicinity of Mott transitions. Thus, it is important to clarify the interrelationship between SC and a Mott transition.

The purpose of this paper is to understand simultaneously SC and a Mott transition arising in the Hubbard model on an anisotropic triangular lattice at half filling, with $\kappa$-ET salts in mind. To this end, we use a variational Monte Carlo (VMC) method, which is very useful in studying the physics of intermediate correlation strength as a continuous function of $U / t .^{19}$ In the variational wave functions, we introduce a factor controlling the binding between a doublon and a holon, which is necessary to appropriately treat the Hubbard model including Mott transitions. ${ }^{20}$ We carefully check the systemsize dependence with highly accurate VMC data; this check is indispensable for the study of phase transitions.

It is found that a first-order Mott transition takes place at $U=U_{\mathrm{c}}$ approximately of the bandwidth for a wide $t^{\prime} / t$ range. The $d$-wave superconducting (SC) correlation function indicates that robust $\mathrm{SC}$ is realized immediately below $U_{\text {c }}$ for a moderate frustration $\left(0.4 \lesssim t^{\prime} / t \lesssim 0.7\right)$. For weakly frustrated cases $\left(t^{\prime} / t \lesssim 0.4\right)$, the SC is defeated by an AF state with a long-range order; for highly frustrated cases $\left(t^{\prime} / t \gtrsim 0.8\right)$, the SC correlation becomes weak for arbitrary values of $U / t$, together with a decrease in the degree of AF correlation. Thus, the origin of the $\mathrm{SC}$ can be attributed to the AF correlation.

*E-mail: h042203d@mbox.nagoya-u.ac.jp 
(a)

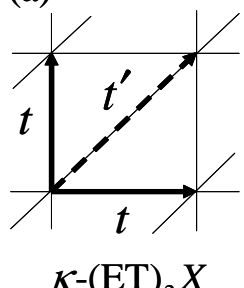

(b)

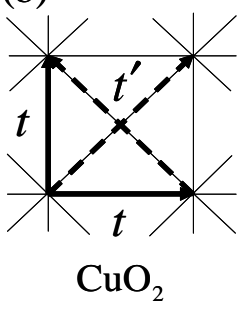

Fig. 1. Lattice structure and hopping integrals of models (a) used in this study for $\kappa$-ET salts, and (b) often used for high- $T_{\mathrm{c}}$ cuprates. The latter is shown for comparison.

The organization of this paper is as follows: In $\S 2$, the model and method used are introduced. Sections 3-5 are assigned to the description of our results and comparisons with other theories. Section 3 treats the behavior of condensation energies of the $d$-wave and AF states. In $\S 4$, we demonstrate that a Mott transition actually takes place in the present approach by studying various quantities, and reveal the properties of this Mott transition. In $\S 5$, we describe robust $d$-wave $\mathrm{SC}$ and its relevance to the AF spin correlation. $\S 6$ presents further discussions; first we compare our results with those of related experiments on $\kappa$-ET salts, followed by an analysis of the Mott insulating state in light of strong-coupling theories. In $\S 7$, we summarize our results. In Appendix A, we point out the limitation of Gutzwiller-type approximations in dealing with a Mott transition. In Appendix $\mathrm{B}$, we comment on the related previous variational studies in the following context. Although a couple of studies in the early $90^{\prime} \mathrm{s}^{21,22}$ concluded that a Mott transition never arises in such wave functions as treated in this paper, this conclusion has already been proven incorrect. ${ }^{20}$ However, a recent VMC study on the present subject ${ }^{23}$ has still followed the above wrong conclusion and made some misinterpretations.

Part of this study has been reported in a proceedings. $^{24}$

\section{Model and Method}

According to the extended Hückel calculations based on the structure of $\kappa$-ET salts, ${ }^{3,25}$ their complicated conducting planes are described with an anisotropic triangular lattice, whose unit cell is composed of a dimer of ET molecules. In each dimer (or unit cell), the strong hybridization between two ET molecules leads to a large split of the levels of bonding and antibonding orbitals. The antibonding orbital accommodates one electron, and composes a conduction band of half filling. Because the Coulomb interaction is relatively strong due to the slight overlap between $\pi$ orbitals, a Hubbard model has been considered as a realistic model. ${ }^{5,26}$

We hence consider a single-band Hubbard model on an anisotropic triangular lattice [see Fig.1(a)] given as

$$
\begin{array}{r}
\mathcal{H}=\mathcal{H}_{\text {kin }}+\mathcal{H}_{\text {int }}=\sum_{\mathbf{k} \sigma} \varepsilon_{\mathbf{k}} c_{\mathbf{k} \sigma}^{\dagger} c_{\mathbf{k} \sigma}+U \sum_{i} n_{i \uparrow} n_{i \downarrow}, \\
\varepsilon_{\mathbf{k}}=-2 t\left(\cos k_{x}+\cos k_{y}\right)-2 t^{\prime} \cos \left(k_{x}+k_{y}\right),
\end{array}
$$
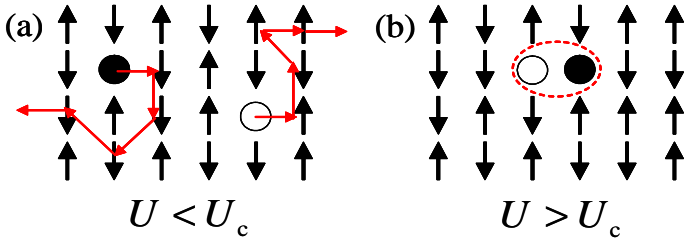

Fig. 2. (Color online) Schematic explanation of a Mott transition due to binding of a doublon to a holon described by $\Psi_{Q}^{d}$. The solid (open) circles denote doublons (holons). (a) In a conductive regime, a doublon and a holon (charge carriers) can move independently. (b) In an insulating regime, a doublon and a holon are confined within adjacent sites; thereby, charge cannot flow. The results of Mott transitions are treated in $\S 4$.

with $U, t, t^{\prime}>0 .{ }^{27}$ Throughout this paper, we fix the electron density at half filling $\left(n=N_{\mathrm{e}} / N_{\mathrm{s}}=1 ; N_{\mathrm{e}}\right.$ : electron number, $N_{\mathrm{s}}$ : site number).

To this model, we apply an optimization VMC method $^{28}$ that can correctly treat the local correlation in the whole parameter space spanned by $U / t$ and $t^{\prime} / t$. As a variational wave function, we use a Jastrow type: $\Psi=\mathcal{P} \Phi$, in which $\Phi$ is a one-body (Hartree-Fock) part expressed as a Slater determinant, and $\mathcal{P}$ is a many-body correlation factor. Concerning $\mathcal{P}$, the onsite (Gutzwiller) factor $^{29}$

$$
\mathcal{P}_{\mathrm{G}}=\prod_{i}\left[1-(1-g) n_{i \uparrow} n_{i \downarrow}\right],
$$

was found effective for $t$ - $J$-type models. ${ }^{30,31}$ For the Hubbard model, however, it has been known ${ }^{32}$ that the wave functions $\mathcal{P}_{\mathrm{G}} \Phi\left(=\Psi_{\mathrm{G}}\right)$ yield several unfavorable results, especially for large values of $U / t$ : (1) The momentum distribution function $n(\mathbf{k})$ is an increasing function of $|\mathbf{k}|$. (2) $2 k_{\mathrm{F}}$ anomalies in the spin [charge density] structure factor $S(\mathbf{q})[N(\mathbf{q})]$ cannot be properly represented. (3) A Mott transition cannot be described, in addition to a sizably high variational energy.

To overcome these flaws, one has to introduce an intersite correlation factor. ${ }^{21,33}$ In particular, at half filling, the doublon-holon binding factor $\mathcal{P}_{Q},{ }^{20,21,34}$ in addition to $\mathcal{P}_{\mathrm{G}}$, is an indispensable element for describing a Mott transition. Generally, $\mathcal{P}_{Q}$ should be written as a functional of $\mu(\mathbf{r})$, which is a function of the relative position of a doubly occupied site (doublon) from an empty site (holon), $\mathbf{r}{ }^{21}$ Here, however, we integrate only the two major elements into $\mathcal{P}_{Q}$, namely, for nearest neighbors, $\mu[\mathbf{r}=( \pm x, 0)$ and $(0, \pm y)]$ and for second (diagonal) neighbors, $\mu^{\prime}[\mathbf{r}=(x, y)$ and $(-x,-y)]$.

$$
\begin{gathered}
\mathcal{P}_{Q}=\prod_{i}\left(1-\mu Q_{i}^{\tau}\right)\left(1-\mu^{\prime} Q_{i}^{\tau^{\prime}}\right) \\
Q_{i}^{\tau\left(\tau^{\prime}\right)}=\prod_{\tau\left(\tau^{\prime}\right)}\left[d_{i}\left(1-e_{i+\tau\left(\tau^{\prime}\right)}\right)+e_{i}\left(1-d_{i+\tau\left(\tau^{\prime}\right)}\right)\right]
\end{gathered}
$$

Here, $d_{i}=n_{i \uparrow} n_{i \downarrow}, e_{i}=\left(1-n_{i \uparrow}\right)\left(1-n_{i \downarrow}\right)$, and $\tau\left(\tau^{\prime}\right)$ runs over all adjacent sites in the bond directions of $t\left(t^{\prime}\right)$. Our trial wave function thus becomes

$$
\Psi_{Q}=\mathcal{P}_{Q} \mathcal{P}_{\mathrm{G}} \Phi .
$$

The factor $\mathcal{P}_{Q}$ is naturally derived by taking account of virtual states in the second-order processes in the 
strong-coupling expansion, ${ }^{35,36}$ and controls the binding strength between a doublon and a holon with the parameters $\mu$ and $\mu^{\prime}\left(\mu, \mu^{\prime} \leq 1\right)$. For $\mu\left(\mu^{\prime}\right) \sim 0$, doublons and holons, which are charged, move around almost freely, whereas for $\mu\left(\mu^{\prime}\right) \rightarrow 1$, a doublon and a holon are tightly bound within adjacent sites in the bond directions of $t$ $\left(t^{\prime}\right)$ (See Fig. 2). Thus, current cannot flow for $\mu \rightarrow 1$. It has been argued in detail ${ }^{20}$ that this factor $\mathcal{P}_{Q}$ can describe a Mott transition and its existence has been actually confirmed for a regular square lattice. Afterward, Mott transitions have been found using $\mathcal{P}_{Q}$ for various systems (refer to $\S$ B.1 in Appendix B). ${ }^{19,37-40}$

Regarding $\Phi$, we treat three cases in this paper, a $d$ wave BCS state, an AF state and a Fermi sea. First, we introduce a singlet wave function of the form of the BCS function with a $d_{x^{2}-y^{2}}$-wave gap

$$
\begin{gathered}
\Phi_{d}=\left(\sum_{\mathbf{k}} \varphi_{\mathbf{k}} c_{\mathbf{k} \uparrow}^{\dagger} c_{-\mathbf{k} \downarrow}^{\dagger}\right)^{\frac{N_{\mathrm{e}}}{2}}|0\rangle, \\
\varphi_{\mathbf{k}}=\frac{\Delta_{\mathbf{k}}}{\tilde{\varepsilon}_{\mathbf{k}}-\zeta+\sqrt{\left(\tilde{\varepsilon}_{\mathbf{k}}-\zeta\right)^{2}+\Delta_{\mathbf{k}}^{2}}} .
\end{gathered}
$$

with

$$
\tilde{\varepsilon}_{\mathbf{k}}=-2 t\left(\cos k_{x}+\cos k_{y}\right)-2 \tilde{t}^{\prime} \cos \left(k_{x}+k_{y}\right),
$$

and $\Delta_{\mathbf{k}}=\Delta_{d}\left(\cos k_{x}-\cos k_{y}\right)$. Here, $\zeta, \Delta_{d}$, and $\tilde{t}^{\prime}$ are variational parameters to be optimized and $\zeta$ corresponds to the chemical potential. Although the parameter $\Delta_{d}$ indicates the magnitude of the $d$-wave gap, it does not necessarily mean the stability of SC; in the insulating phase, $\Delta_{d}$ is related to the spin gap. Furthermore, we take account of a renormalization effect of the quasi-Fermi surface due to the electron correlation. The main part of this effect is introduced into $\Phi_{d}$ by optimizing $\tilde{t}^{\prime} / t$, independently of $t^{\prime} / t$ given in the Hamiltonian eq. (1). ${ }^{41}$ As we will see later, $\tilde{t}^{\prime} / t$ notably deviates from the original $t^{\prime} / t$, particularly in the insulating phase, and the renormalization affects the stability of the $d$-wave singlet state.

Next, as a competing state for weakly frustrated (small- $\left.t^{\prime} / t\right)$ cases, we consider the commensurate AF long-range ordered state

$$
\Phi_{\mathrm{AF}}=\prod_{\mathbf{k}, \sigma}\left(\tilde{u}_{\mathbf{k}} c_{\mathbf{k} \sigma}^{\dagger}+\operatorname{sgn}(\sigma) \tilde{v}_{\mathbf{k}} c_{\mathbf{k}+\mathbf{K} \sigma}^{\dagger}\right)|0\rangle,
$$

with

$$
\tilde{u}_{\mathbf{k}}\left(\tilde{v}_{\mathbf{k}}\right)=\sqrt{\frac{1}{2}\left(1-(+) \frac{\varepsilon_{\mathbf{k}}}{\sqrt{\varepsilon_{\mathbf{k}}^{2}+\Delta_{\mathrm{AF}}^{2}}}\right)} .
$$

Here, $\mathbf{K}=(\pi, \pi)$, and the wave-number space $(\mathbf{k})$ is restricted to the folded AF Brillouin zone. $\Delta_{\mathrm{AF}}$ is a variational parameter indicating the magnitude of the $\mathrm{AF}$ gap. At half filling, $\Psi_{\mathrm{AF}}\left(=\mathcal{P}_{Q} \mathcal{P}_{\mathrm{G}} \Phi_{\mathrm{AF}}\right)$ with finite $\Delta_{\mathrm{AF}}$ is always insulating, as in the mean-field theory.

Lastly, as a reference state, we use a projected Fermi sea: $\Psi_{\mathrm{F}}\left(=\mathcal{P}_{Q} \mathcal{P}_{\mathrm{G}} \Phi_{\mathrm{F}}\right)$ with $\Phi_{\mathrm{F}}=\prod_{|\mathbf{k}|<k_{\mathrm{F}} \sigma} c_{\mathbf{k} \sigma}^{\dagger}|0\rangle$. Although $\Psi_{\mathrm{F}}$ also brings about a Mott transition at a somewhat larger $U$ than the bandwidth, ${ }^{20,37}$ we will not treat the insulating regime of $\Psi_{\mathrm{F}}$ in this paper, and thus call it the 'normal' or 'metallic' state.

In the remainder of this section, we briefly mention the details of VMC calculations. Because the trial functions we treat have at most six parameters, we employ a simple linear optimization of each parameter with the other parameters fixed. In a round of iteration, every parameter is once optimized in one dimension. In almost all the cases of optimization in this study, the parameters converge within five rounds, after which we continue the optimization for more than ten rounds. The optimized values of the parameters and energy are determined by averaging the results of the ten rounds after convergence. Because the optimization is carried out with $2-5 \times 10^{5}$ samples, our data are substantially averages of several million samples. Thus, we can greatly suppress the statistical errors in energy down to the order of $10^{-4} t$. Such precision is necessary to correctly analyze the physics in the vicinity of a phase transition. Physical quantities are calculated with the optimized parameters thus obtained with $2.5 \times 10^{5}$ samples.

The systems used are of $N_{\mathrm{s}}=L \times L$ sites with periodicantiperiodic boundary conditions. A closed shell condition is always imposed. We mainly study the systems for $L=10$ and 12 , and check those for $L=14$ and 16 when we would like to know the system-size dependence more definitely. As we will see later, for $t^{\prime} \neq 0$, in contrast to for $t^{\prime}=0$, the system-size dependence is not monotonic; in particular, the $\mathrm{SC}$ correlation function is very sensitive to the configuration of occupied $k$ points. Thus, we need to deduce the properties for $L \rightarrow \infty$ from the tendency of finite-sized systems in varying $L$.

\section{Stability of $d$-wave and AF states}

In this and the following two sections, we describe results of VMC calculations, and compare with other theoretical ones. In this section, we first consider the competition between $d$-wave and AF states, and then mention the critical behavior found for $\Psi_{Q}^{d}$.

To consider the competition between $\Psi_{Q}^{\mathrm{AF}}$ and $\Psi_{Q}^{d}$, we take up the condensation energies defined by $E_{\mathrm{c}}^{d}\left(E_{\mathrm{c}}^{\mathrm{AF}}\right)=$ $E^{\mathrm{F}}-E^{d}\left(E^{\mathrm{AF}}\right)$, in which $E^{d}, E^{\mathrm{AF}}$, and $E^{\mathrm{F}}$ are the optimized variational energies per site with respect to $\Psi_{Q}^{d}$, $\Psi_{Q}^{\mathrm{AF}}$ and $\Psi_{Q}^{\mathrm{F}}$, respectively. For $t^{\prime}=0$ (the regular square lattice), it is well-known that the ground state has an AF long-range order for arbitrary positive values of $U / t$ due to the complete nesting condition; this feature can be properly described by the present approach, namely $E_{\mathrm{c}}^{\mathrm{AF}}>E_{\mathrm{c}}^{d}>0 .{ }^{19}$ The maximum $E_{\mathrm{c}}^{\mathrm{AF}}$ is $0.148 t(U / t=8)$ for $L=12$, and will be a little larger for $L \rightarrow \infty .^{37}$

In Fig. 3, we show $E_{\mathrm{c}}^{d}$ and $E_{\mathrm{c}}^{\mathrm{AF}}$ for four values of $t^{\prime} / t$ $(=0.2-0.8)$. As the frustration becomes stronger $\left(t^{\prime} / t\right.$ increases), the magnitude of $E_{\mathrm{c}}^{\mathrm{AF}}$ rapidly decreases, and thereby the stable region of the AF state shrinks. For $t^{\prime} / t=0.2$, the AF region still remains: $U \lesssim 6.7(L=10)$ and $U \lesssim 7.5(L=12)$. For $t^{\prime} / t=0.4$, however, the AF region becomes very narrow for $L=10(5 \lesssim U \lesssim 6.5)$, and finally vanishes for $L=12\left(E_{\mathrm{c}}^{\mathrm{AF}}<E_{\mathrm{c}}^{d}\right)$, as seen in the inset of Fig. 3(b). These indicate that at $t^{\prime} / t \sim 0.4$, the ground state switches from the AF state to the $d$ - 

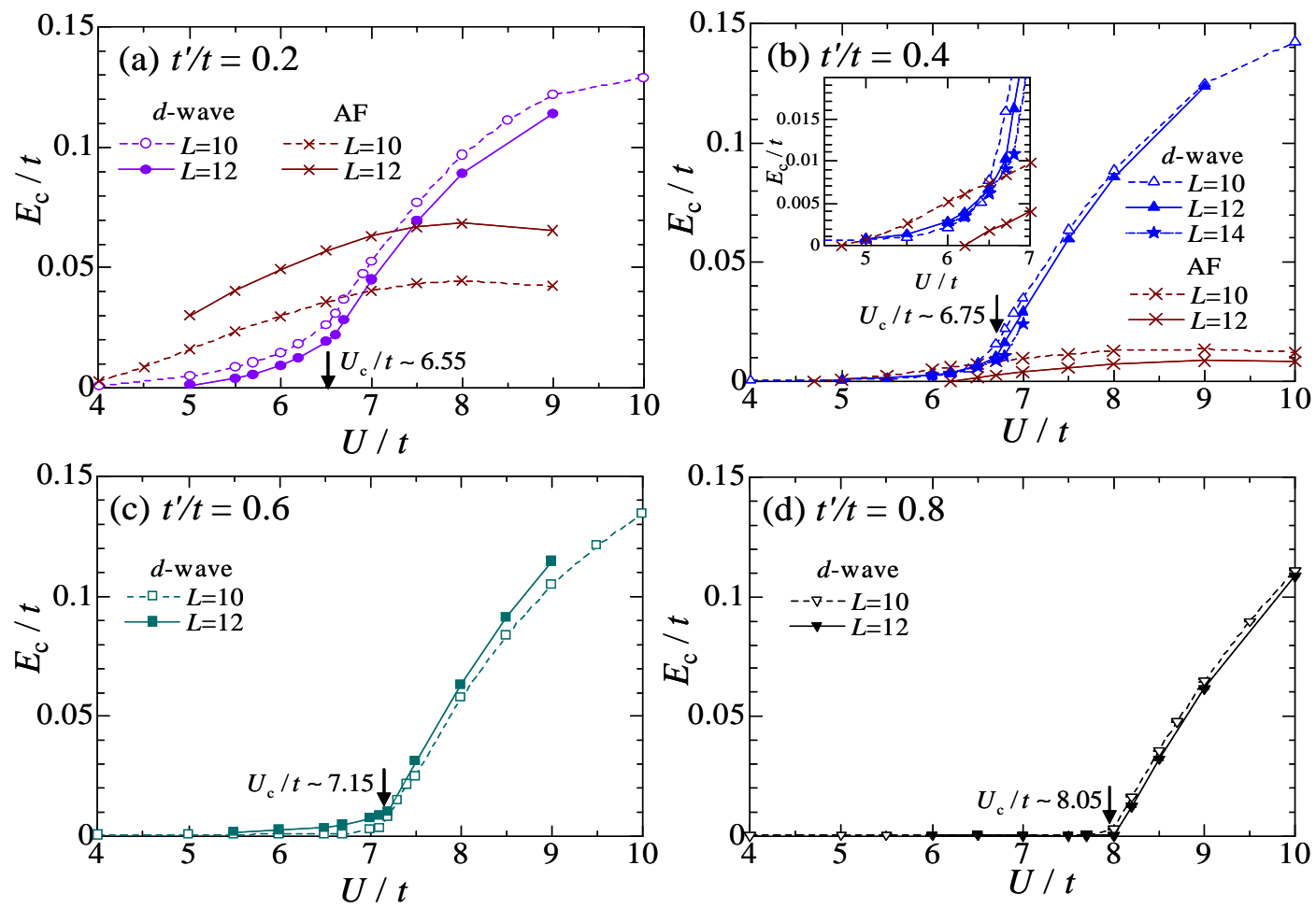

Fig. 3. (Color online) Condensation energies $E_{\mathrm{c}} / t$ of $d$-wave and AF states for four values of $t^{\prime} / t$ : (a) $t^{\prime} / t=0.2$, (b) $0.4,(\mathrm{c}) 0.6$, and (d) 0.8 . For $t^{\prime} / t=0.6$ and $0.8, E_{\mathrm{c}}^{\mathrm{AF}}$ is zero for arbitrary values of $U / t$. The arrow in each panel indicates the critical point, $U_{\mathrm{c}} / t$ of the Mott transition arising in the $d$-wave state. The values of $U_{\mathrm{c}} / t$ shown are estimated from the quantities treated in $\S 4$ and $\S 5$. The inset in (b) is a close-up near the critical point. Systems of mainly $L=10-12$ are compared.

wave state, although the system-size dependence of $E_{\mathrm{c}}^{\mathrm{AF}}$ is not simple near $t^{\prime} / t=0.4$ (see $m_{\mathrm{s}}$ in Fig. 13). For $t^{\prime} / t=$ 0.6 and 0.8 , the AF state is never stabilized with respect to the normal state. This feature is natural at half filling, because the nesting of the Fermi surface monotonically becomes worse, as $t^{\prime} / t$ increases.

In the following, we consider the behavior of $E_{\mathrm{c}}^{d}$. For small values of $U / t(\lesssim 4), E_{\mathrm{c}}^{d}$ is very small for arbitrary values of $t^{\prime} / t$, indicating that $\mathrm{SC}$ is fragile, if any. $E_{\mathrm{c}}^{d}$ gradually increases for intermediate values of $U / t$ : $4 \lesssim U / t \lesssim 6.5$ for $t^{\prime} / t=0.2,5.5 \lesssim U / t \lesssim 6.5$ for $t^{\prime} / t=0.4$, and $6 \lesssim U / t \lesssim 7$ for $t^{\prime} / t=0.6$. For $t^{\prime} / t=0.8$, this gradual increase is hardly seen. As will be shown in $\S 5$, this increase corresponds to the stabilization due to robust SC. For every $t^{\prime} / t, E_{\mathrm{c}}^{d}$ suddenly and markedly increases at $U=U_{\mathrm{c}}$, which is indicated by arrows in Fig. 3 . This increase in $E_{\mathrm{c}}^{d}$ certainly indicates the stabilization of the $d$-wave singlet state, but $\Psi_{Q}^{d}$ is not necessarily a $\mathrm{SC}$ state, as explained in $\S 2$. We will pursue this behavior in the next section.

\section{Mott transitions}

In this section, we first demonstrate that the critical behavior found in $\Psi_{Q}^{d}$ in $\S 3$ is attributed to a Mott transition by studying various quantities (§4.1), and then reveal the properties of the transition from different points of view $(\S 4.2)$.

\subsection{Confirmation of Mott transitions}

First, we consider the optimized values of the variational parameters in $\Psi_{Q}^{d}$, which are plotted in Fig. 4 for four values of $t^{\prime} / t$. For each $t^{\prime} / t$, all the variational parameters have discontinuities at the same $U / t$, which is identical to $U_{\mathrm{c}} / t$ mentioned in the preceding section. Here, $U_{\mathrm{c}}$ is determined accurately: $U_{\mathrm{c}} / t=$ $6.55,6.75,7.15$, and 8.05 for $t^{\prime} / t=0.2,0.4,0.6$, and 0.8 , respectively. These discontinuities indicate that some first order phase transition takes place at $U=U_{\mathrm{c}}$. Actually, near $U=U_{\mathrm{c}}, E^{d}$ has double minima in the parameter space; a hysteresis in $E^{d}$ is observed, as to whether we approach $U_{\mathrm{c}}$ from the small- $U$ side or the large- $U$ side, and particularly notable in the cases of large $t^{\prime} / t$. Here, recall that the doublon-holon binding factor $\mu$ is a good index of a Mott transition. In the small- $U$ regime, $\mu$ is as small as 0.1-0.3 and almost independent of $L$, whereas $\mu$ is likely to approach 1 for $U>U_{\mathrm{c}}$, in allowing for the large $L$ dependence [Fig. 4(d)]. Thus, this transition is expected to be a Mott transition. In the following, we will confirm this expectation with various quantities.

Let us start with the momentum distribution function

$$
n(\mathbf{k})=\frac{1}{2 N_{\mathrm{s}}} \sum_{k, \sigma}\left\langle c_{\mathbf{k} \sigma}^{\dagger} c_{\mathbf{k} \sigma}\right\rangle .
$$

Shown in Fig. 5 is $n(\mathbf{k})$ measured with the optimized parameters along the path $(0,0)-(\pi, 0)-(\pi, \pi)-(0,0)$ for $t^{\prime} / t=0.4$ and 0.8 . Because $\Psi_{Q}^{d}$ has a node of the gap in the $(0,0)-(\pi, \pi)$ direction, metallic properties should develop in this direction; $n(\mathbf{k})$ should have a discontinu- 

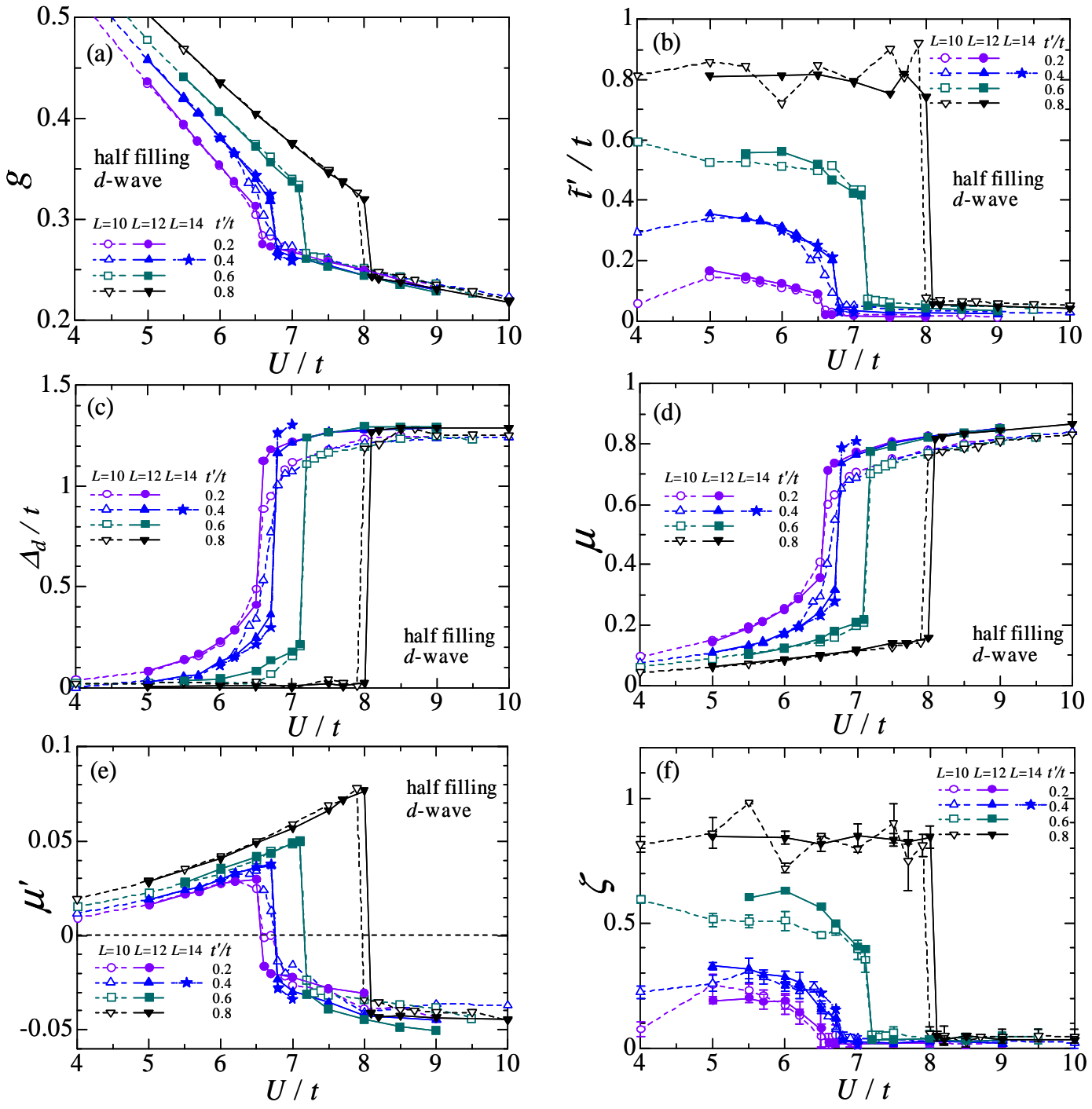

Fig. 4. (Color online) Optimized values of variational parameters in $d$-wave singlet state, $\Psi_{Q}^{d}$, for four values of $t^{\prime} / t$ as functions of $U / t$; (a) $g$ [onsite (Gutzwiller) factor], (b) $\tilde{t}^{\prime} / t$ [band renormalization factor], (c) $\Delta_{d} / t$ [ $d$-wave gap], (d) $\mu$ [doublon-holon binding factor in the direction of $t$ ], (e) $\mu^{\prime}$ [the same of $t^{\prime}$ ], (e) $\zeta$ [chemical potential]. The systems are of $L=10-14$. Statistical errors in $\tilde{t}^{\prime} / t$ and in $\zeta$ increase as $U / t$ decreases or $t^{\prime} / t$ increases, due to the discrete energy levels or $k$ points.

ity at $k_{\mathrm{F}}$. For both values of $t^{\prime} / t$, the discontinuity at $k_{\mathrm{F}}$ is apparent for $U<U_{\mathrm{c}}$, whereas, for $U>U_{\mathrm{c}}$, the discontinuity, that is, the quasi-Fermi surface disappears. Thus, metallic properties are abruptly lost at $U=U_{\mathrm{c}}$ even in the nodal direction of the $d_{x^{2}-y^{2}}$ wave.

To see this feature quantitatively, the quasi-particle renormalization factor $Z$ is a suitable indicator, which roughly corresponds to the inverse of effective mass, unless the $k$-dependent renormalization of self energy is severe. In Fig. 6 , we plot $Z$ estimated from the magnitude of jump in $n(\mathbf{k})$ at $\mathbf{k}=\mathbf{k}_{\mathrm{F}}$ in the nodal direction. ${ }^{42}$ For all the values of $t^{\prime} / t, Z$ decreases slowly for $U<U_{\mathrm{c}}$, whereas, at $U=U_{\mathrm{c}}, Z$ suddenly vanishes with a sizable discontinuity, reflecting the first-order character of the transition. The system-size dependence of $Z$ is weak, and the magnitude of $Z$ tends to increase as $L$ increases $\left(t^{\prime} / t=0\right)$. The behavior of $Z$ strongly suggests that the effective electron mass diverges for $U>U_{\mathrm{c}}$. Incidentally, the discontinuity in $Z$ at $U=U_{\mathrm{c}}$ is a feature that dif- fers from those of $\Psi_{Q}^{\mathrm{F} 19,20}$ and of a dynamical mean-field theory for the hypercubic lattice, ${ }^{43}$ in which $Z$ gradually decreases and vanishes at $U=U_{\text {c }}$ without a jump. Thus, the first-order character of the transition is more conspicuous in $\Psi_{Q}^{d}$.

Next, we consider the charge structure factor

$$
N(\mathbf{q})=\frac{1}{N_{\mathrm{s}}} \sum_{i, j} e^{i \mathbf{q} \cdot\left(\mathbf{R}_{i}-\mathbf{R}_{j}\right)}\left\langle N_{i} N_{j}\right\rangle-n^{2},
$$

with $N_{i}=n_{i \uparrow}+n_{i \downarrow}$. Within the variational theory, $N(\mathbf{q}) \propto|\mathbf{q}|$ for $|\mathbf{q}| \rightarrow 0$, if the state does not have a gap in the charge degree of freedom, whereas $N(\mathbf{q}) \propto \mathbf{q}^{2}$, if a charge gap opens. In Fig. $7, N(\mathbf{q})$ is depicted for two different values of $t^{\prime} / t(0.4$ and 0.8$)$ near $U=U_{\text {c }}$. For both values of $t^{\prime} / t, N(\mathbf{q})$ near the $\Gamma$ point $(0,0)$ seems linear in $|\mathbf{q}|$ for $U<U_{\mathrm{c}}$ (solid symbols), whereas it abruptly changes to being roughly quadratic in $|\mathbf{q}|$ for $U>U_{\text {c }}$ (open symbols). It follows that $\Psi_{Q}^{d}$ is gapless in the charge sector and is conductive for $U<U_{\mathrm{c}}$, but a 

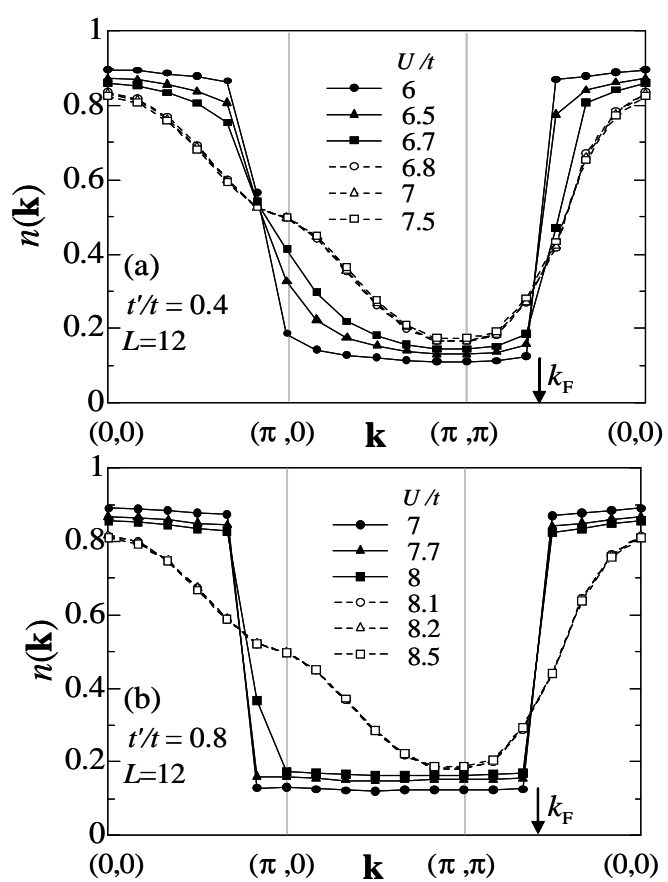

Fig. 5. Momentum distribution function $n(\mathbf{k})$ of $d$-wave SC state for (a) $t^{\prime} / t=0.4\left(U_{\mathrm{c}} / t \sim 6.75\right)$ and (b) $0.8\left(U_{\mathrm{c}} / t \sim 8.05\right)$. The solid (open) symbols denote the points of $U<U_{\mathrm{c}}\left(U>U_{\mathrm{c}}\right)$. The arrows indicate the positions of quasi-Fermi surface in the node-of-gap direction $(0,0)-(\pi, \pi)$. The systems are of $L=12$.

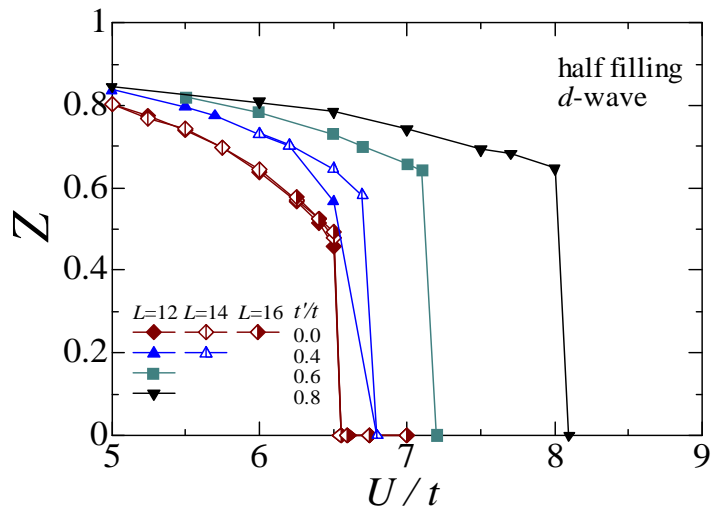

Fig. 6. (Color online) Quasiparticle renormalization factor $Z$ in node-of-gap direction of $d$-wave singlet states for four values of $t^{\prime} / t$ as a function of $U / t$. The deviation of the data for $t^{\prime} / t=0.4$, $L=12$ and $U / t=6.5$ is a special case, and is ascribed to the discrete $k$ points of the system. ${ }^{42}$

charge gap opens for $U>U_{\mathrm{c}}$ and $\Psi_{Q}^{d}$ becomes insulating.

As further evidence for a Mott transition, we consider the doublon density (density of doubly-occupied sites),

$$
D=\frac{1}{N_{\mathrm{s}}} \sum_{i} n_{i \uparrow} n_{i \downarrow}=\frac{1}{N_{\mathrm{s}}} \frac{\left\langle\mathcal{H}_{\mathrm{int}}\right\rangle}{U}
$$

which is regarded as the order parameter of Mott transitions, ${ }^{44}$ by analogy with the particle density in gas-liquid transitions. In Fig. 8, we plot $D$ for several values of $t^{\prime} / t$. As the interaction strength $U / t$ increases, the magnitude of $D$ decreases almost linearly in $U / t$ for small $U / t$, but abruptly drops at $U_{\mathrm{c}} / t$, and then decreases slowly for
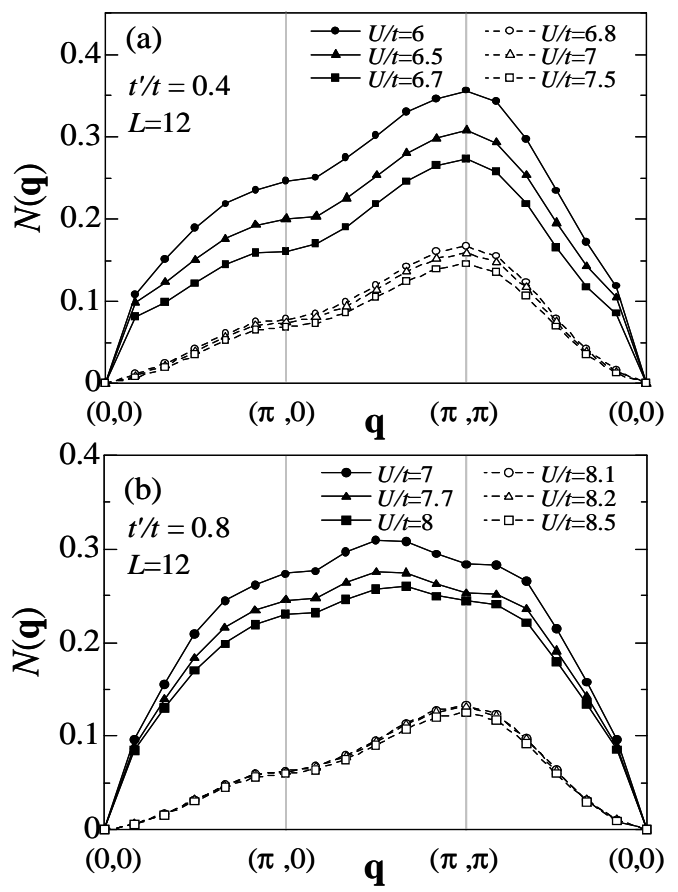

Fig. 7. Charge structure factor $N(\mathbf{q})$ of $d$-wave singlet state for (a) $t^{\prime} / t=0.4$ and (b) 0.8 . Solid (open) symbols indicate the data for $U<U_{\mathrm{c}}\left(U>U_{\mathrm{c}}\right)$. The systems are of $L=12$.

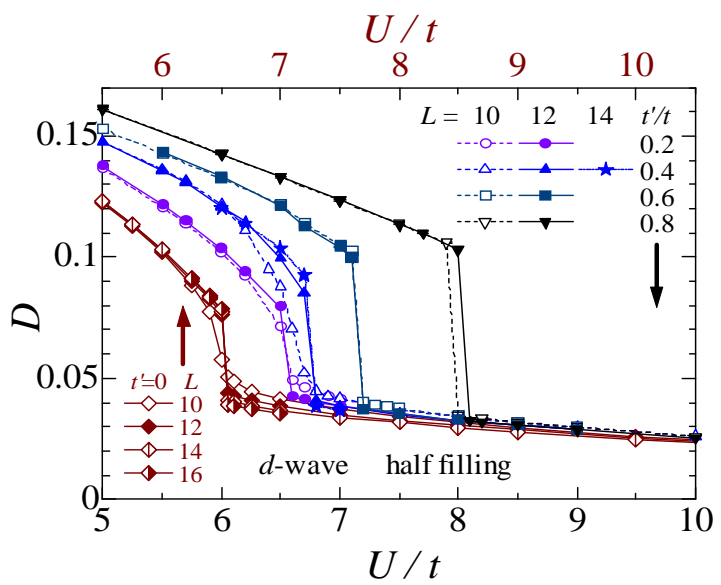

Fig. 8. (Color online) Density of doublon (doubly occupied site) of $d$-wave singlet state as a function of $U / t$ near $U=U_{\mathrm{c}}$ for four values of $t^{\prime} / t$. For $t^{\prime} / t=0$, the horizontal axis is shifted by 0.5 (see upper axis) for clarity. This quantity is reduced to $0.25(0)$ in the limit of $U / t \rightarrow 0(\infty)$ at half filling. Data for some system sizes are shown for each $t^{\prime} / t$.

$U>U_{\mathrm{c}} \cdot{ }^{45}$ This behavior of $D$ corroborates the idea of a first-order Mott transition.

Owing to the behavior of all the quantities studied above, we can safely judge that a Mott transition takes place in the $d$-wave singlet state at $U=U_{\text {c }}$ for arbitrary values of $t^{\prime} / t$.

\subsection{Properties of Mott transitions}

For a start, we contrast this transition with the wellknown theory of Brinkman and Rice, ${ }^{46}$ which is based 
on $\Psi_{\mathrm{G}}$. Their theory is a milestone in the research on Mott transitions, and still survives as a symbolic concept of a certain class of transitions. However, it should be noted that the result of their approximation additionally applied, the so-called Gutzwiller approximation ${ }^{49}$ (GA), has been proven correct only in infinite space dimensions. In accurate calculations of $\Psi_{\mathrm{G}},{ }^{20,32,47,48}$ a metalinsulator transition is absent in finite dimensions. We sum up the properties of the Brinkman-Rice transition. For a half-filled-band Hubbard model, as $U / t$ increases toward $U=U_{\mathrm{BR}}=8 E_{0}\left(E_{0}\right.$ : energy for $\left.U=0\right)$, electrons are gradually localized in lattice sites one by one, and the magnetic susceptibility diverges as $U \rightarrow U_{\mathrm{BR}}$; this transition is a continuous type. For $U>U_{\mathrm{BR}}$, electrons are completely localized and never move, namely $E_{\text {kin }}=E_{\text {int }}=0$. Hence, the behavior of $|E| \sim 4 t^{2} / U$ $(=J)$ in the insulating regime cannot be derived from this framework.

In contrast, in the present theory, the Mott transition is described not by the disappearance of charge carriers, but by the binding (and unbinding) of negative carriers (doublons) to positive carriers (holons), as explained for $\mathcal{P}_{Q}$ in $\S 2$ and schematically shown in Fig. 2 . In this mechanism, doublons (and holons) never vanish even for $U>U_{\text {c }}$, as we can actually observe $g>0$ in Fig. 4(a) and $D>0$ in Fig. 8. The process of creating a temporary pair of a doublon and a holon through the so-called virtual hopping leads to the behavior proportional to $t^{2} / U$.

In this connection, a couple of studies ${ }^{50,51}$ have recently addressed the present subject, applying GA for $d$-wave BCS states $^{52}$ to Hubbard-type models with extra exchange-coupling terms. We compare the results of these studies with those of conventional GA for the Fermi sea in Appendix A. As long as the Mott transition is concerned, the results of GA for the $d$-wave BCS state have features qualitatively different from the present VMC results, and share the behavior with the conventional GA for the Fermi sea.

Next, we consider the function of $\mu^{\prime}$. For $U<U_{\mathrm{c}}$, $\mu^{\prime}$ gradually increases, as $U / t$ increases, similarly to $\mu$, although $\left|\mu^{\prime}\right|$ is small compared with $|\mu|[$ Figs. $4(\mathrm{~d})$ and $4(\mathrm{e})]$. Thus, $\mu^{\prime}$ plays an analogous role to $\mu$ in the conductive phase. Concerning the $t^{\prime} / t$ dependence, the behavior of $\mu^{\prime}$ is opposite to that of $\mu$, as naturally expected. At $U=U_{\mathrm{c}}$, however, $\mu^{\prime}$ suddenly drops to a negative value, irrespective of $t^{\prime} / t$. This behavior means that, in the diagonal direction, a doublon and a holon as two independent particles are more favorable than those as a bound pair in the neighbors. As we will demonstrate shortly, this is probably because the AF short-range correlation noticeably develops for $U \gtrsim U_{\mathrm{c}}$; thereby, parallel spin configurations are favored in the diagonal directions, and suppress the hopping in terms of $t^{\prime}$. This marked direction dependence of $\mu(\mathbf{r})$ is a new feature specific to two dimensions, in particular, to the $d$-wave singlet state. According to the exact-diagonalization calculations for onedimensional systems, the behavior of $\mu(r)$ for a large $U / t$ is simple, namely, $\mu(r)$ is approximately proportional to $\exp \left(-r / r_{0}\right)$, in which $r_{0}$ is a constant. ${ }^{21}$

In what follows, we consider the $t^{\prime} / t$ dependence of this Mott transition in some points. In Fig. 9, the crit-

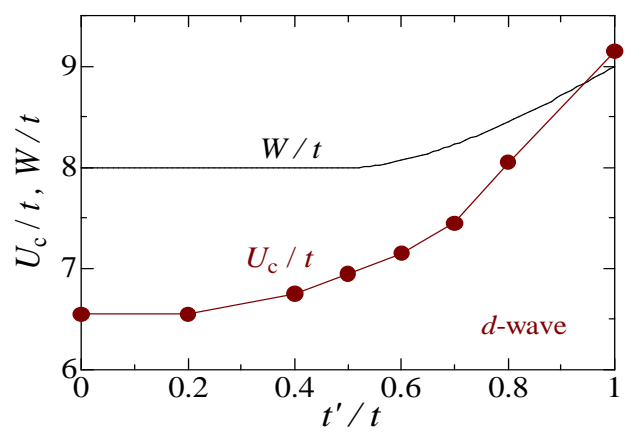

Fig. 9. (Color online) Comparison between bare bandwidth $W$ and critical value $U_{\mathrm{c}}$ of Mott transition for $d$-wave singlet state. Because $U_{\mathrm{c}} / t$ is determined using finite-sized data (mainly $L=$ 12 ), the critical values for $L=\infty$ may somewhat shift to the larger side of $U / t$, especially for large values of $t^{\prime} / t$.

ical value $U_{\mathrm{c}}$ is compared with the bare bandwidth $W$. For the present dispersion eq. (2), $W$ is constant, $8 t$, for $0 \leq t^{\prime} / t \leq 0.5$, and gradually increases as $t^{\prime} / t$ increases over 0.5 . As $W / t$ increases, the absolute value of kinetic energy increases, leading to a situation advantageous for the conductive state. Consequently, $U_{\mathrm{c}} / t$ increases with $W / t$; actually, $U_{\mathrm{c}} / t$ starts to increase at a smaller $t^{\prime} / t$ than $W / t$. This feature is common to different Motttransition systems. The ratio $U_{\mathrm{c}} / W$ has become a topic in some studies; both dynamical mean field calculations for infinite-dimensional lattices, ${ }^{53}$ and VMC calculations using $\Psi_{Q}^{\mathrm{F} 20,37}$ for $t^{\prime} / t=0$ yield values of $U_{\mathrm{c}} / W$ somewhat larger than 1 . In this work, however, $U_{\mathrm{c}} / W=0.81$ for $t^{\prime} / t=0$, and increases with $t^{\prime} / t$ toward $1.02(L=12)$ for $t^{\prime} / t=1.0$, which is relatively close to the result of DCA, $1.15-1.2$, for $t^{\prime} / t=1 .^{17}$ Conversely, this ratio obtained using PIRG is rather small, $U_{\mathrm{c}} / W=0.58$, even for $t^{\prime} / t=1.0 .^{18}$

Now, we turn to the order of the Mott transition. Returning to Fig. 4, we notice that the discontinuity at $U=U_{\mathrm{c}}$ increases, as $t^{\prime} / t$ increases, in every parameter. Such a tendency is common to other quantities like $Z$ (Fig. 6) and $D$ (Fig. 8), and is also observed in the hysteresis or double-minimum structure in $E^{d}$, which becomes more conspicuous, as $t^{\prime} / t$ increases (not shown). This indicates that the character of a first-order transition becomes prominent, as the frustration becomes stronger $\left(t^{\prime} / t\right.$ increases $)$. This feature seems unique to $\Psi_{Q}^{d}$, and in contrast to that of the Mott transition in $\Psi_{Q}^{\mathrm{F}},{ }^{37}$ as well as that of PIRG. ${ }^{54}$ In these cases, the effect of frustration makes the transitions more like those of the continuous type. This characteristic behavior of $\Psi_{Q}^{d}$ is probably caused by the prominent stability in the insulating regime, studied in the following and $§ 6.2$.

In the remainder of this subsection, we consider the spin degree of freedom of the Mott insulating state described by $\Psi_{Q}^{d}$ for $U>U_{\mathrm{c}}$. Shown in Fig. 10 is the spin structure factor,

$$
S(\mathbf{q})=\frac{1}{N_{\mathrm{s}}} \sum_{i j} e^{i \mathbf{q} \cdot\left(\mathbf{R}_{i}-\mathbf{R}_{j}\right)}\left\langle S_{i}^{z} S_{j}^{z}\right\rangle,
$$

for two values of $t^{\prime} / t$. Leaving the behavior in the conductive regime for the next section, here we focus on the 


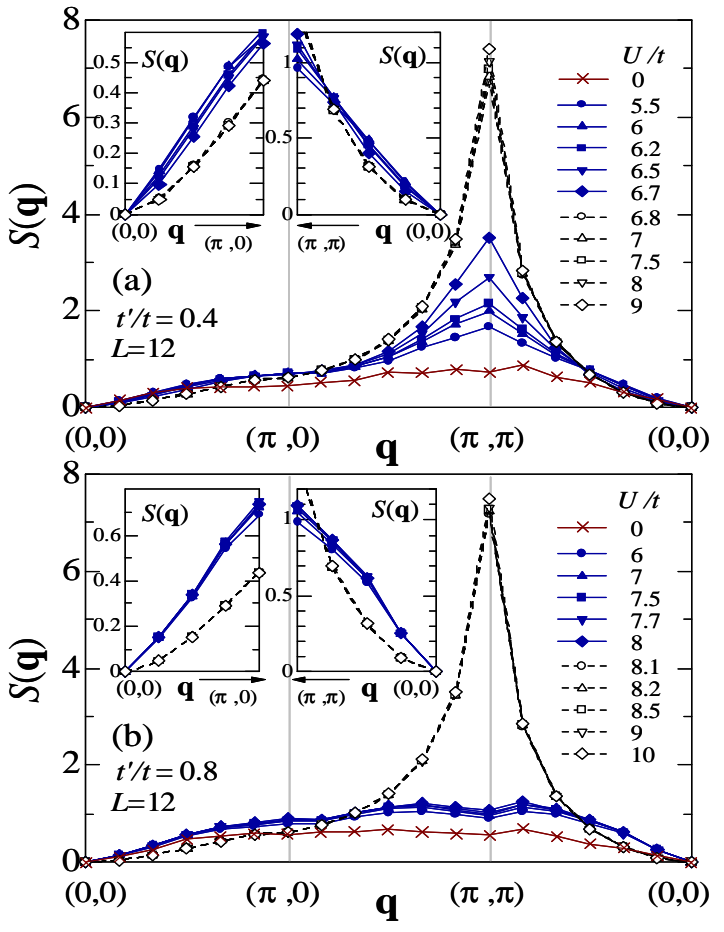

Fig. 10. (Color online) Spin structure factor $S(\mathbf{q})$ of $d$-wave singlet state along the same path as in Fig. 5 are compared between (a) $t^{\prime} / t=0.4$ and (b) 0.8 . Solid (open) symbols denote the points belonging to the conductive (insulating) region. The insets in each panel are close-ups near the $\Gamma$ point $(0,0)$ in the directions of $(0,0)-(\pi, 0)$ (left) and $(0,0)-(\pi, \pi)$ (right). The systems are of $L=12$.

insulating state shown by open symbols. For $U>U_{\mathrm{c}}$, $S(\mathbf{q})$ has a prominent peak at $\mathbf{q}=\mathbf{K}=(\pi, \pi)$, compared with that for $U<U_{\mathrm{c}}$; the AF spin correlation markedly develops in the insulating phase. On the other hand, the height of peaks, $S(\mathbf{K})$, has only weak system-size dependence compared with $L$ as well as $L^{2}$ : For example for $t^{\prime} / t=0.4$ and $U / t=7, S(\mathbf{K})=6.36,6.85$, and 7.28 for $L=10,12$, and 14 , respectively. In the same sense, the staggered magnetization

$$
m_{\mathrm{s}}=\frac{1}{N_{\mathrm{s}}}\left|\sum_{i}(-1)^{\left|\mathbf{R}_{i}\right|}\left\langle S_{i}^{z}\right\rangle\right|
$$

with $S_{i}^{z}=\left(n_{i \uparrow}-n_{i \downarrow}\right) / 2,{ }^{55}$ is always zero for $\Psi_{Q}^{d}$ within the statistical fluctuation. Thus, this insulating state has sizable short-range AF correlation, although a long-range order is not formed. The enhancement of AF correlation reflects the change in some quantities. In Fig. 4(b), where the renormalized value of the diagonal hopping integral is plotted versus $U / t$, we find that $\tilde{t}^{\prime} / t$ suddenly drops to very small values at $U=U_{\mathrm{c}}$, irrespective of the model value $t^{\prime} / t$. Therefore, the quasi-Fermi surface recovers the nesting condition to a considerable extent, as shown in Fig. 11(c). This feature was also reported in a study using DCA. ${ }^{17}$ Moreover, the chemical potential $\zeta$ becomes almost zero [Fig. 4(f)], namely, the value of the regular square lattice $\left(t^{\prime}=0\right)$, for which the nesting condition is fully satisfied.

The short-range nature of the AF spin correlation affects the low-energy spin excitation. In the insets of
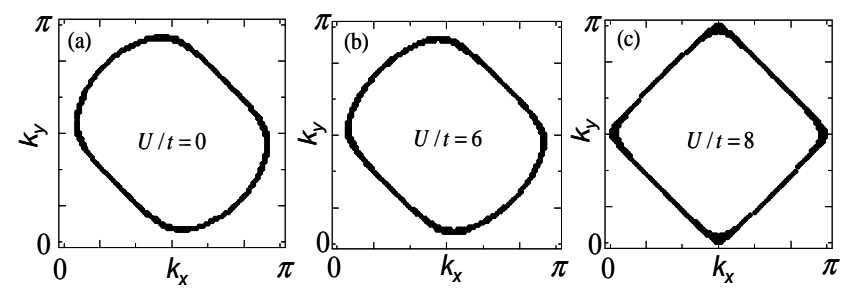

Fig. 11. Effective quasi-Fermi surfaces for (a) $U / t=0$, (b) 6 (SC phase) and (c) 8 (insulating phase). Here, the model value $t^{\prime} / t$ is fixed at 0.4 , whereas the optimized value of $\tilde{t}^{\prime} / t$ in the wave function is renormalized as $\tilde{t}^{\prime} / t=0.31$ (0.02) for $U / t=6$ (8).

Fig. 10, we depict the magnifications of $S(\mathbf{q})$ near the $\Gamma$ point. For $U>U_{\mathrm{c}}, S(\mathbf{q})$ is not proportional to $|\mathbf{q}|$ for $|\mathbf{q}| \rightarrow 0$ but to $|\mathbf{q}|^{2}$ in both bond and diagonal directions and in both values of $t^{\prime} / t$, indicating that this insulating state has a gap in the spin degree of freedom.

In $\S 6.2$, this insulating state will be considered again in light of strong coupling theories.

\section{Superconductivity and phase diagram}

In this section, we elucidate where, in the parameter space, the $d$-wave SC is dominant ( $\$ 5.1)$. Therefore, we construct a phase diagram of the present model. We also study the properties of the $\mathrm{SC}$, emphasizing the relevance to the $\mathrm{AF}$ spin correlation $(\S 5.2)$.

\subsection{Range of robust superconductivity}

As an indicator of SC with the $d_{x^{2}-y^{2}}$ symmetry, it is convenient for the present approach to study the $d$-wave nearest-neighbor pair correlation function ${ }^{56}$ defined as

$$
\begin{aligned}
P_{d}(\mathbf{r})= & \frac{1}{4 N_{\mathrm{s}}} \sum_{i} \sum_{\tau, \tau^{\prime}=\hat{\mathbf{x}}, \hat{\mathbf{y}}}(-1)^{1-\delta\left(\tau, \tau^{\prime}\right)} \times \\
& \left\langle\Delta_{\tau}^{\dagger}\left(\mathbf{R}_{i}\right) \Delta_{\tau^{\prime}}\left(\mathbf{R}_{i}+\mathbf{r}\right)\right\rangle,
\end{aligned}
$$

in which $\hat{\mathbf{x}}$ and $\hat{\mathbf{y}}$ denote the lattice vectors in the $x$ and $y$-directions, respectively, and $\Delta_{\tau}^{\dagger}\left(\mathbf{R}_{i}\right)$ is the creation operator of a nearest-neighbor singlet expressed as

$$
\Delta_{\tau}^{\dagger}\left(\mathbf{R}_{i}\right)=\left(c_{i \uparrow}^{\dagger} c_{i+\tau \downarrow}^{\dagger}+c_{i+\tau \uparrow}^{\dagger} c_{i \downarrow}^{\dagger}\right) / \sqrt{2} .
$$

If $P_{d}(\mathbf{r})$ has a finite value for $|\mathbf{r}| \rightarrow \infty$, one can judge that an off-diagonal long-range order is realized. However, for finite systems, especially, in the cases with small $U / t$, where the correlation length is large, we have to measure long-distance values of $P_{d}(\mathbf{r})$ appropriately. In this paper, we average $P_{d}(\mathbf{r})$ 's with $\mathbf{r}$ being on the line segments $(0, L / 2)-(L, L / 2)$ and $(L / 2,0)-(L / 2, L)$, which correspond to the furthermost edges from the origin, for weak correlation regimes. ${ }^{57}$ For strong correlation regimes, $P_{d}(\mathbf{r})$ becomes almost constant for $|\mathbf{r}|>3 ;^{55}$ we average $P_{d}(\mathbf{r})$ 's with $|\mathbf{r}|>3$ in these cases.

In Fig. 12, we plot the averages of $P_{d}(\mathbf{r})$ thus obtained $\left(P_{d}^{\text {ave }}\right)$ as a function of $U / t$ for four values of $t^{\prime} / t$. The data for $t^{\prime} / t=0.7$ are plotted in Fig. B·2(b). First, let us consider features common to the cases of weak and intermediate frustrations $\left(t^{\prime} / t=0.2-0.7\right)$. For small $U / t$, the magnitude of $P_{d}^{\text {ave }}$ is as small as that for the noninteracting case $(U / t=0)$; here, robust SC cannot be expected. $P_{d}^{\text {ave }}$ starts to increase appreciably at a value 

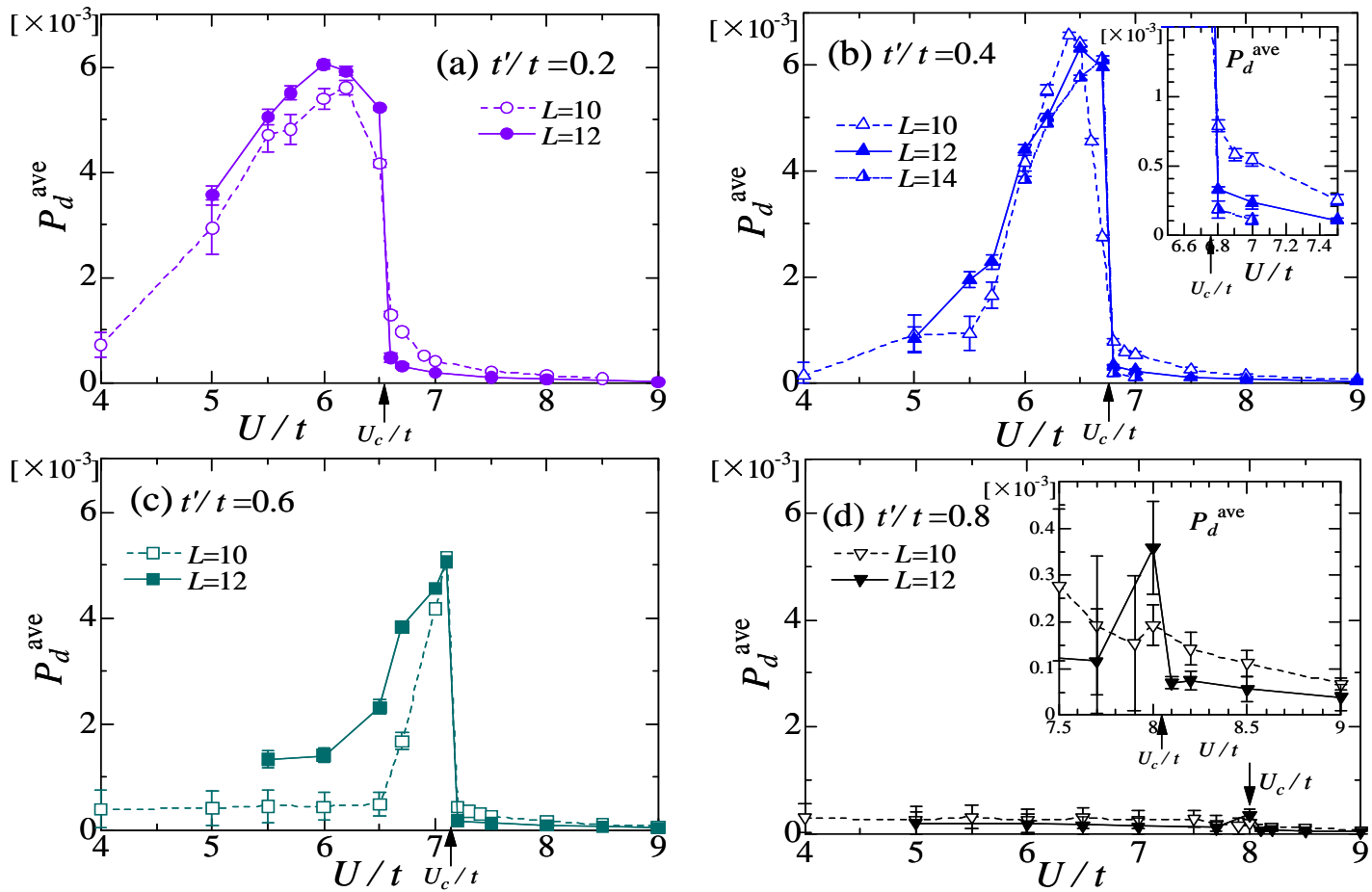

Fig. 12. (Color online) Real-space SC correlation function for nearest-neighbor pairs of $d$-wave symmetry for (a) $t^{\prime} / t=0.2$, (b) 0.4 , (c) 0.6, and (d) 0.8. Plotted are the averages of the long-distance part of $P_{d}(\mathbf{r})$ explained in the text. The insets in (b) and (d) are close-ups near $U_{\mathrm{c}} / t$. Data of different system sizes $(L=10-14)$ are compared. The same quantity for $t^{\prime} / t=0.7$ is shown in Fig. B.2(b) in Appendix.

of $U / t\left(U_{\text {onset }} / t\right)$, which is different for a different $t^{\prime} / t$. There is a peak in $P_{d}^{\text {ave }}$ immediately below $U_{\mathrm{c}}$. The fact that the magnitude of $P_{d}^{\text {ave }}$ near the peak is almost independent of system size indicates that a region of robust SC exists immediately below the Mott critical point $U_{\mathrm{c}}$. At $U_{\mathrm{c}} / t, P_{d}^{\text {ave }}$ suddenly drops to a very small value, which is justifiably expected from the Mott transition. In the insulating regime $\left(U>U_{\mathrm{c}}\right)$, as the system size $L$ increases, the magnitude of $P_{d}^{\text {ave }}$ rapidly decreases as seen in every panel in Fig. 12, and probably vanishes in the limit of $L \rightarrow \infty$. In the insulating regime, the statistical fluctuation in the VMC data is much smaller than that in the conductive regime; the disappearance of $P_{d}(\mathbf{r})$ for $U>U_{\text {c }}$ is absolutely certain. In $\S \mathrm{B} .2$, we comment on a recent VMC study, ${ }^{23}$ which has drawn contradictory conclusions from these results.

Next, we proceed to the $t^{\prime} / t$-dependence of $P_{d}^{\text {ave }}$. As the strength of frustration $t^{\prime} / t$ increases, the onset $U_{\text {onset }} / t$, at which $P_{d}^{\text {ave }}$ starts to increase appreciably, increases: $U_{\text {onset }} / t \sim 4\left(t^{\prime} / t=0.2\right), \sim 5(0.4), \sim 6$ $(0.6)$, and $\sim 7$ (0.7). Because, compared with the increasing rate of $U_{\text {onset }} / t$, that of $U_{\mathrm{c}} / t$ is slow: $6.55(0.2)$, $6.75(0.4), 7.15(0.6)$, and $7.45(0.7)$, the range in $U / t$ of the dominant SC becomes narrower, as $t^{\prime} / t$ increases, as observed in Fig. 12. In Fig. 13, we depict the maximal $P_{d}^{\text {ave }}$ with $t^{\prime} / t$ fixed and $U / t$ varied. This value is almost constant for $t^{\prime} / t \leq 0.4$, decreases as $t^{\prime} / t$ increases beyond $t^{\prime} / t=0.4$, and finally vanishes at approximately $t^{\prime} / t=0.8$. Actually for a strongly frustrated case $\left(t^{\prime} / t=0.8\right)$, an enhancement in $P_{d}^{\text {ave }}$ is hardly seen

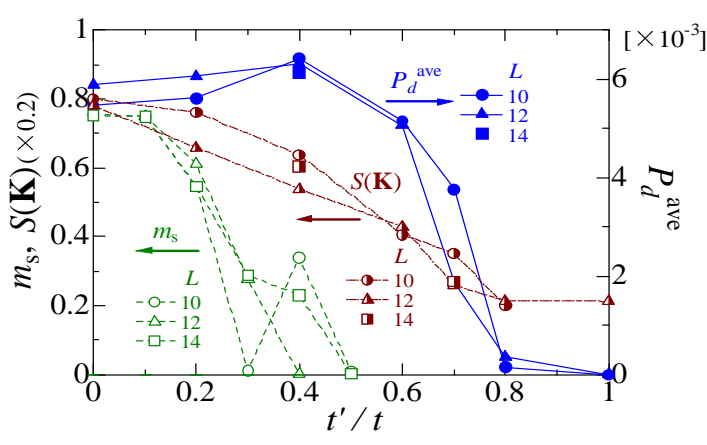

Fig. 13. (Color online) Comparison between the maximal values of $P_{d}^{\text {ave }}$ (average of the SC correlation function) for fixed values of $t^{\prime} / t$ (with $U / t$ changed) and $S(\mathbf{K})$ (spin structure factor at the $\mathrm{AF}$ wave number) calculated with the same parameter of $P_{d}^{\text {ave }}$. Also shown is the sublattice magnetization $m_{\mathrm{s}}$ calculated using $\Psi_{Q}^{\mathrm{AF}}$ at $U / t=6.0$. The scatter of data points, in particular, near the critical values of $t^{\prime} / t$, is ascribed to finite system sizes. Statistical errors are much smaller.

even below $U_{\mathrm{c}} / t(=8.05)$, as in Fig. 12(d), in which the magnitude of $P_{d}^{\text {ave }}$ obtained for $U / t=4-8$ is comparable to the statistical fluctuation. Thus, stable SC is absent from the strongly frustrated (or nearly isotropic) region: $t^{\prime} / t \gtrsim 0.8$. Now, recall that the $\mathrm{AF}$ state with a long-range order is more stable than the SC state for $t^{\prime} / t \lesssim 0.4$, as demonstrated in $\S 3$. This feature is reflected in the sublattice magnetization $m_{\mathrm{s}}[\mathrm{eq} .(16)]$ for $\Psi_{Q}^{\mathrm{AF}}$, as shown in Fig. 13. Thus, we estimate the range of stable $\mathrm{SC}$ as $0.4 \lesssim t^{\prime} / t \lesssim 0.7$. 
Incidentally, as shown in the insets of Fig. 10(a), $S(\mathbf{q})$ tends to be proportional to $|\mathbf{q}|^{2}$ for $|\mathbf{q}| \rightarrow 0$ as $U / t$ increases $\left(U<U_{\mathrm{c}}\right)$, indicating a spin gap is apt to open as SC develops. In contrast, in the insets of Fig. 10(b), $S(\mathbf{q})$ remains linear in $|\mathbf{q}|$, even if $U / t$ increases; $\Psi_{Q}^{d}$ remains metallic for $t^{\prime} / t=0.8$, even if $U$ approaches $U_{\mathrm{c}}$.

The present results for SC are consistent, even quantitatively, with those of FLEX. ${ }^{6,7}$ According to Kino and Kontani, the AF order defeats SC for $t^{\prime} / t<0.4$, whereas the region of finite $T_{\mathrm{c}}$ of $d_{x^{2}-y^{2}}$-wave $\mathrm{SC}$ appears for $0.4<t^{\prime} / t<0.8$ with the highest values taken for $t^{\prime} / t=0.5-0.6$. Finally, $T_{\mathrm{c}}$ of SC disappears for $t^{\prime} / t>0.8$. Furthermore, the range of SC in $U / t$ displays a tendency similar to that of the present study.

Having discussed the range of SC in the $t^{\prime}-U$ space and its stability, let us now reconsider how various quantities behave in this SC region. First, concerning the condensation energy shown in Fig. 3, we are now aware that the gradual increase in $E_{\mathrm{c}}$ for $U_{\text {onset }} \lesssim U<U_{\mathrm{c}}$ derives from SC, whereas the sizable gain for $U>U_{\mathrm{c}}$ is not due to SC but to the $d$-wave singlet spin state. Similar gradual changes can be seen in the variational parameters (Fig. 4); in particular, the increase in $\Delta_{d}$ for $U_{\text {onset }} \lesssim U<U_{\text {c }}$ directly corresponds to the development of SC [Fig. 4(c)]. The slow decrease in $\tilde{t}^{\prime} / t$ [Fig. 4(b)] means that the degree of renormalization of $\tilde{\varepsilon}_{\mathbf{k}}$ is low in the SC state; the quasi-Fermi surface is not very different from that for $U / t=0$ as in Figs. 11(a) and (b). This tendency is again consistent with the result of FLEX. ${ }^{6}$ Note that such a gradual change never appears in any quantity in the case of $t^{\prime} / t=0.8$, where robust $\mathrm{SC}$ is absent.

\subsection{Properties of superconductivity}

Because the SC develops as soon as the AF long-range order disappears, the AF correlation must be important for the mechanism of SC. We hence begin with the spin structure factor $S(\mathbf{q})$ [eq. (15)], returning to Fig. 10. For $U / t=0, S(\mathbf{q})$ does not have a peak but rather a dip at the $\mathrm{AF}$ wave number $\mathbf{K}=(\pi, \pi)$ for both $t^{\prime} / t=0.4$ and 0.8 , due to the frustration. In the weakly frustrated case $\left(t^{\prime} / t=0.4\right)$, in which robust SC appears, $S(\mathbf{K})$ steadily grows as $U / t$ increases, and $\mathbf{K}$ becomes a characteristic wave number even in the conductive regime, $U<U_{\mathrm{c}}$ [solid symbols in Fig. 10(a)]. In contrast, in the strongly frustrated case $\left(t^{\prime} / t=0.8\right)$, in which the SC correlation does not develop, although $S(\mathbf{q})$ somewhat increases as a whole for $U<U_{\mathrm{c}}$ [solid symbols in Fig. 10(b)], preserving the shape for $U / t=0$, yet $S(\mathbf{q})$ does not exhibit a special enhancement at $\mathbf{q}=\mathbf{K}$, but has maxima at incommensurate wave numbers. To check this point, we plot, in Fig. 13, $S(\mathbf{K})$ for $\Psi_{Q}^{d}$ giving the maximal $P_{d}^{\text {ave }}$; when $S(\mathbf{K})$ decreases, the maximal $P_{d}^{\text {ave }}$ simultaneously decreases. We have confirmed it for a wide range of model parameters that whenever $P_{d}^{\text {ave }}$ is appreciably enhanced, $S(\mathbf{q})$ has an evident peak at $\mathbf{q}=\mathbf{K}$. From these discussions, we can conclude that $\mathrm{SC}$ in this model is induced by the AF spin correlation. Thus, we can understand that the shape of the quasi-Fermi surface of $\Psi_{Q}^{d}$ tends to be more square-lattice-like $\left(\tilde{t}^{\prime} / t^{\prime}<1\right)$, as mentioned earlier,

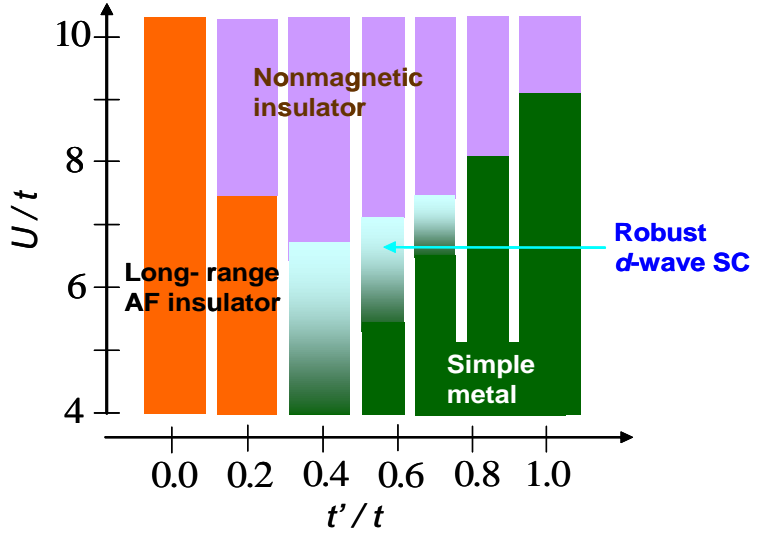

Fig. 14. (Color online) Phase diagram in the $t^{\prime}-U$ plane constructed from the present VMC results.

so as to place the nesting vector $\mathbf{K}$ on hot spots having a larger density of state. It is probable that the mechanism of SC proposed here is basically the same as those of the weak-coupling theories, ${ }^{6,7,58}$ and is also common to that of high- $T_{\mathrm{c}}$ cuprates at half filling, if any, which have quite similar lattice structure (see Fig. 1). ${ }^{37}$

Here, we summarize the features of this SC in light of the AF correlation. (1) For small values of $U / t$, because magnetic correlations do not fully develop, robust SC does not appear. (2) For small values of $t^{\prime} / t$, although the $\mathrm{AF}$ correlation develops as $U / t$ increases, an $\mathrm{AF}$ longrange order defeats $\mathrm{SC}$ due to the nesting condition. (3) For moderate values of $t^{\prime} / t$ as well as $U / t$, the AF correlation is not strong enough to form an AF long-range order, but the short-range part survives. Thereby, stable $d$-wave SC is realized. (4) For large values of $t^{\prime} / t$, even short-range AF correlation decays due to severe frustration. Thus, SC is not likely to arise. (5) For considerably large values of $U / t$, a Mott insulating state suppresses SC.

On the basis of the results mentioned upto this point, we constructed a phase diagram in the model space spanned by $t^{\prime} / t$ and $U / t$ (Fig. 14). Robust $d$-wave SC appears in the highlighted area $\left(0.4 \lesssim t^{\prime} / t \lesssim 0.7\right.$, $U_{\text {onset }} \lesssim U<U_{\text {c }}$ ), which is in contact with both $\mathrm{AF}$ and Mott insulating areas, and continues to the metallic area in the small- $U / t$ or large- $t^{\prime} / t$ sides, where the $\mathrm{SC}$ correlation is very weak. The conductive (metallic or SC) and nonmagnetic insulating areas are divided by a firstorder Mott transition described within the $d$-wave singlet state. The boundary between the areas of long-range AF insulator and the nonmagnetic insulator (or superconductor) is determined by comparing the energies of $\Psi_{Q}^{\mathrm{AF}}$ and $\Psi_{Q}^{d}$.

Finally, we take up another weak-coupling feature of this SC. Shown in Fig. 15 are the components of condensation energy, that is, the differences in kinetic and interaction energies between $\Psi_{Q}^{d}$ and $\Psi_{Q}^{\mathrm{F}}$ given as

$$
\begin{aligned}
\Delta E_{\mathrm{kin}}^{d} & =E_{\mathrm{kin}}^{\mathrm{F}}-E_{\mathrm{kin}}^{d}, \\
\Delta E_{\mathrm{int}}^{d} & =E_{\mathrm{int}}^{\mathrm{F}}-E_{\mathrm{int}}^{d},
\end{aligned}
$$

in which $\Delta E_{\text {kin }}^{d}+\Delta E_{\text {int }}^{d}=E_{\mathrm{c}}^{d}(\geq 0)$. In the SC regime $\left(U<U_{\mathrm{c}}\right), \Delta E_{\mathrm{int}}^{d}\left(\Delta E_{\mathrm{kin}}^{d}\right)$ is always positive (negative), 

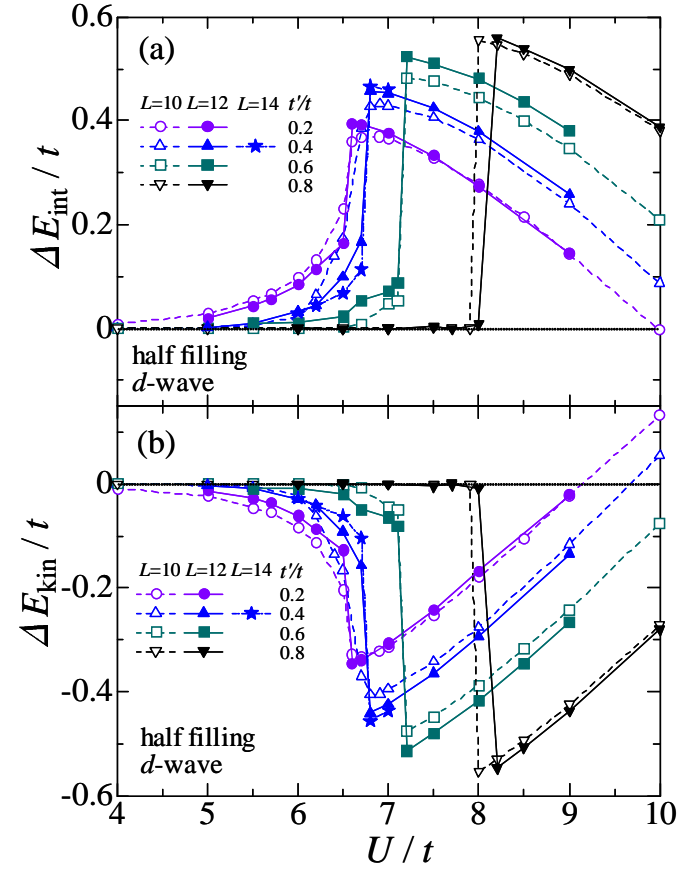

Fig. 15. (Color online) Differences in (a) interaction and (b) kinetic energies between normal and $d$-wave states for $t^{\prime} / t=0.2$, $0.4,0.6$, and 0.8 .

regardless of $t^{\prime} / t$. This indicates that the SC transition is induced by the gain in interaction energy at the cost of kinetic energy. This feature smoothly continues to the weak-coupling limit $(U / t \rightarrow 0)$, and common to conventional BCS superconductors. Although the component of energy gain switches to the kinetic energy for $U \gtrsim 10 t$, as seen in Fig. 15, yet a SC phase is not realized for $U>U_{\mathrm{c}}$; the SC due to the kinetic-energy gain is not realized here, in contrast to the doped cases. ${ }^{19}$

\section{Further Discussions}

In $\S 6.1$, we compare the results obtained in this work with those of related experiments on $\kappa$-ET salts. In $\S 6.2$, as a continuation of $\S 4.2$, we analyze $\Psi_{Q}^{d}$ for $U>U_{\mathrm{c}}$ in light of strong-coupling theories, ${ }^{59}$ and propose a couple of improvements.

\subsection{Comparisons with experiments}

First, let us think of SC. If the present results for SC is applicable to $\kappa$-ET salts, the SC arising in them should be induced by AF correlation, which becomes weak as $t^{\prime} / t$ increases (Fig. 13). Actually, in a class of $\kappa$-ET salts, a compound with weaker frustration (smaller $t^{\prime} / t$ ) tends to have higher $T_{\mathrm{c}}$ : for $\mathrm{X}=\mathrm{CuN}(\mathrm{CN})_{2} \mathrm{Br}\left(t^{\prime} / t=0.68\right)$, $\mathrm{Cu}(\mathrm{NCS})_{2}(0.84)$, and $\mathrm{Cu}_{2}(\mathrm{CN})_{3}(1.06), T_{\mathrm{c}}=11.6,10.4$, and $3.9 \mathrm{~K}$ (under pressure of $0.4 \mathrm{GPa}$ ), respectively. Note, however, that, according to our result, robust SC does not arise for $t^{\prime} / t \gtrsim 0.8$; this fact seemingly contradicts the experiments on $\kappa$-ET salts, particularly of the compound $\mathrm{X}=\mathrm{Cu}_{2}(\mathrm{CN})_{3}$. A point we must not overlook here is the effect of pressure. The compound $\mathrm{X}=\mathrm{Cu}_{2}(\mathrm{CN})_{3}$ has an almost regular triangular structure $\left(t^{\prime}=t\right)$ under ambient pressure, which fact is also confirmed by a hightemperature-expansion study. ${ }^{60}$ However, this compound does not exhibit SC, unless a pressure of $0.4-0.6 \mathrm{GPa}$ is applied. ${ }^{61}$ It is well-known that applied pressure generally enhances the bandwidth (reduce $U / t$ ). It is reasonable to expect that $t^{\prime} / t$, in addition to $U / t$, should be greatly varied by pressure, ${ }^{62}$ if we allow for the fact that organic compounds are soft and anisotropic, and the values of $t$ and $t^{\prime}$ are sensitive to bond angle. Actually, a recent NMR- $T_{1}$ experiment for $\mathrm{X}=\mathrm{Cu}_{2}(\mathrm{CN})_{3}{ }^{61}$ has suggested the enhancement of AF fluctuation under pressure; in this case, it is likely that $t^{\prime} / t$ is sufficiently reduced to induce SC. Although the pressure effect on $t^{\prime} / t$ is quite an important problem, it has not yet been established whether $t^{\prime} / t$ is reduced ${ }^{63}$ or enhanced $^{64}$ by pressure.

Next, we touch on Mott (SC-nonmagnetic insulator) transition. The existence of a first-order Mott transition is consistent with the experiment on temperaturedependent magnetic susceptibility and the nuclear spinlattice relaxation rate of $\kappa$ - $(\mathrm{ET})_{2} \mathrm{Cu}_{2}(\mathrm{CN})_{3} \cdot{ }^{4}$ In this compound, however, the ground state in the insulating phase has a low-energy spin excitation; the spin gap will be very small, if it exists. This point is not necessarily consistent with the present result. Furthermore, the insulating states of most $\kappa$-ET salts exhibit a long-range AF order, although they are considered strongly frustrated with $t^{\prime} / t \gtrsim 0.6$. Even if we take account of the fact that actual materials possess the effect of three dimensions, the trial function in the Mott insulating regime leaves room for improvement with regard to the spin correlation, as will be mentioned in the next subsection. In relation to the insulating state, it is also an interesting future problem how the 120 degree Néel order, which is realized for $U / t \rightarrow \infty$ and $t^{\prime} / t \sim 1$, depends on $U / t$ and $t^{\prime} / t^{65}$

\section{2 $\Psi_{Q}^{d}$ as an RVB state}

Although $\Psi_{Q}^{d}$ has succeeded in describing a Mott transition for an intermediate coupling strength, some adjustments in the spin correlation seem to be needed for the insulating regime $\left(U>U_{\mathrm{c}}\right)$, where a strong-correlation scheme is convenient.

As a previous study for the square lattice showed, ${ }^{19}$ the strong-coupling regime ( $U>U_{\mathrm{c}}$ for half filling) of the Hubbard model shares the properties with $t$-J-type models; the physics adiabatically connected to the limit of $U / t \rightarrow \infty$. This picture also holds in the insulating state here, because the energies are scaled with $t^{2} / U$, and various quantities obtained with $\Psi_{Q}^{d}$ changes very slowly as a function of $U / t$ and $t^{\prime} / t$, as observed in $n(\mathbf{k})$ [Fig. 5], $N(\mathbf{q})$ [Fig. 7], $D$ [Fig. 8] and $S(\mathbf{q})$ [Fig. 10]. In this sense, $\Psi_{Q}^{d}$ is substantially equivalent to Anderson's RVB state. ${ }^{66}$

For $U / t \rightarrow \infty$, the present Hubbard model eq. (1) is reduced to the $J-J^{\prime}$ Heisenberg model with the same connectivity. Some approximations ${ }^{67}$ to this spin model concluded that the Néel order persists up to $J^{\prime} / J=0.6-0.7$ $\left(t^{\prime} / t=0.77-0.84\right)$ beyond the classical value $J^{\prime} / J=0.5$ $\left(t^{\prime} / t=0.71\right)$. In contrast, in this study, the phase bound- 
ary between the AF state and the insulating state of $\Psi_{Q}^{d}$ for $U \rightarrow \infty$ is situated at $t^{\prime} / t<0.2$, as deduced from Fig. 3(a). Thus, the two approaches quantitatively give different estimates. A possible origin of this discrepancy is that, despite the marked $\mathrm{AF}$ short-range correlation, $\Psi_{Q}^{d}$ does not have a seed of AF longer-range correlations. Also, the fact that the renormalization of $t^{\prime} / t$ is not introduced into $\Psi_{Q}^{\mathrm{AF}}$ possibly affects the discrepancy. As an improvement in both $\Psi_{Q}^{d}$ and $\Psi_{Q}^{\mathrm{AF}}$, it is very important to study a wave function that can possess both $d$-wave pairing and AF order. ${ }^{68}$ This will expand the area of AF insulator to some extent (see Fig. 14).

Nonetheless, note that $\Psi_{Q}^{d}$ has an extremely low energy as an insulating state; it readily defeats the AF state even with weak frustration, as in Fig. 3. In this connection, we need to remember Liang et al.'s study, ${ }^{69}$ in which the Heisenberg model on the square lattice is studied with intelligible wave functions, which are composed of a product of singlets with a bond-length distribution as a variational parameter. They showed that the wave functions including long singlet bonds exhibit an AF long-range order with very low energies; the wave functions composed only of short-bond singlets do not have AF long-range order but have very close energies to the former. Thus, the stability of the wave function is little influenced by whether the spin correlation is shortranged or long-ranged. There is no reason to suppose that this situation does not hold for frustrated cases. Because Liang et al.'s wave functions are not tractable for $t^{\prime} / t \neq 0$, we should take an alternative approach, for example, refine a class of projected singlet wave functions by adjusting the gap parameters. They are expressed as the same form as eq. (7) with $\varphi_{\mathbf{k}}$, which generally differs from that of the BCS type, eq. (8), but is tractable in the present scheme.

\section{Summary}

We have studied a Hubbard model on an anisotropic triangular lattice at half filling by an optimization VMC method with adequate precision, taking account of the $\mathrm{SC}$ and Mott transition arising in $\kappa$-ET salts. In the trial functions - normal, $\mathrm{AF}$, and $d$-wave singlet states, we introduce intersite correlation factors that control the binding between a doublon and a holon. We have succeeded in describing the $d$-wave SC and a Mott transition simultaneously in a sole approach, and in accounting for the behavior of a series of $\kappa$-ET salts broadly. We itemize our main results:

(1) Within the $d$-wave singlet state, a first-order Mott (conductor-to-nonmagnetic insulator) transition takes place at $U_{\mathrm{c}}$ approximately of the bandwidth for arbitrary values of $t^{\prime} / t$. The critical value $U_{\mathrm{c}} / t$ gradually increases as band width (or $t^{\prime} / t$ ) increases. This transition is not directly related to a magnetic order.

(2) The nonmagnetic insulating state ( $d$-wave singlet state for $U>U_{\mathrm{c}}$ ) has a considerably low energy and a strong short-range AF correlation. This state has a spin gap, as well as a charge gap.

(3) The AF long-range order prevailing in the weakly frustrated cases $\left(t^{\prime} / t \lesssim 0.4\right)$ is rapidly destabilized as $t^{\prime} / t$ increases, and yields to the $d$-wave singlet state (SC or nonmagnetic insulating).

(4) SC with the $d_{x^{2}-y^{2} \text {-wave symmetry appears for }}$ moderate values of $U / t(\sim 6)$ and $t^{\prime} / t\left(0.4 \lesssim t^{\prime} / t \lesssim 0.7\right)$, whose area is adjacent to both areas of an AF long-range order and a Mott insulator. The phase diagram obtained in this study is shown in Fig. 14.

(5) SC pairs are formed with the aid of the short-range AF spin correlation, which is weakened by the frustration and vanishes for $t^{\prime} / t \gtrsim 0.8$. The SC transition is induced by the gain in interaction energy; this mechanism is identical to that in the weak correlation limit as well as that of conventional BCS superconductors.

In this paper, we have focused on the $d_{x^{2}-y^{2} \text {-wave }}$ symmetry, which is established for $t^{\prime} / t=0 .{ }^{70}$ It has been reported, however, that the symmetry of pairing switches to $d+(i) d$-type symmetries at $t^{\prime} / t=1 .{ }^{23,71,72}$ It is hence worthwhile to check other pairing symmetries for large values of $t^{\prime} / t$. Moreover, it is interesting to check with VMC how the features revealed in the single-band Hubbard model are altered in more realistic multiband models, ${ }^{73}$ especially in strong and intermediate correlation regimes. In connection with pairing symmetry, we finally comment that it is interesting to study the phase sensitive property, e.g., the tunneling effect and Josephson effect, in unconventional superconductor near a Mott transition. ${ }^{14,74}$

\section{Acknowledgments}

The authors thank M. Ogata for useful discussions. They appreciate the discussions with and comments of H. Fukuyama, N. Nagaosa, K. Kanoda, K. Kuroki, and H. Kontani. This work is partly supported by Grant-in-Aids from the Ministry of Education, Culture, Sports, Science and Technology, by NAREGI Nanoscience Project of the same Ministry, by the Supercomputer Center, ISSP, University of Tokyo, and by a Grant-in-Aid for the 21st Century COE "Frontiers of Computational Science".

\section{Appendix A: Gutzwiller approximation for $d$ - wave state}

In this Appendix, we compare the results of conductorinsulator transitions in recent studies ${ }^{50,51}$ using GA for the $d$-wave BCS states $^{52}$ with the previous ones using GA for the Fermi sea. Two groups have recently addressed the present subject by applying GA for $d$-wave BCS states to Hubbard-type models with extra exchangecoupling terms. These extra terms are added for technical reasons, and seem to make no essential difference. Powell and McKenzie ${ }^{51}$ studied a Mott transition and SC without allowing for AF. They found a first-order SC-insulator transition at $U / t$ somewhat larger than the bandwidth. In the insulating regime, however, the kinetic energy vanishes, indicating this transition belongs to the Brinkman-Rice type. The AF effect is incorporated into GA for the $d$-wave BCS state by Gan et al. ${ }^{50}$ who found a SC-AF insulator transition at $U / t$ somewhat smaller than the bandwidth. In the SC regime, the SC correlation and renormalization of $g$ develop as $U / t$ increases without sublattice magnetization, whereas in the AF insulating regime, there appears a finite sublattice magne- 
tization but $g$ is reduced to the value at $U / t=0$, namely $g=1$. In fact, this result is closely parallel to that of the GA for the Fermi sea into which an AF effect is introduced, as in the following.

About three decades ago, Ogawa et al. ${ }^{75}$ introduced the AF effect into the GA formula for the Fermi sea, using $\mathcal{P}_{\mathrm{G}} \Phi_{\mathrm{AF}}$, with a cubic-type lattice in mind. The result obtained exhibits a transition; for $0<U<U_{\mathrm{AF}}$, the result coincides with the ordinary (or paramagnetic) GA formula $\left(\Delta_{\mathrm{AF}}=0, g<1\right)$, whereas for $U>U_{\mathrm{AF}}$, the result is switched to the mean-field solution of AF insulator $\left(\Delta_{\mathrm{AF}}>0, g=1\right)$. Here, $U_{\mathrm{AF}}$ denotes $U$ at which the energy curve of the GA formula intersects with that of the AF mean-field approximation. Thus, the transition at $U=U_{\mathrm{AF}}$ is a first-order AF transition which does not correspond to the Mott transition we considered in this paper, but is basically identical to those derived by the mean-field theories for $t^{\prime} \neq 0 .{ }^{76}$ Later, it is shown by a VMC study ${ }^{77}$ for $\mathcal{P}_{\mathrm{G}} \Phi_{\mathrm{AF}}$ that this conclusion is again incorrect; the true variational state has an $\mathrm{AF}$ gap and a finite renormalization by $g\left(\Delta_{\mathrm{AF}}>0, g<1\right)$ simultaneously for arbitrary values of $U(>0)$, and does not exhibit a transition for $U>0$.

Thus, as long as a Mott transition is concerned, the results of GA for the $d$-wave BCS state $\mathrm{s}^{50,51}$ share the features with the conventional GA for the Fermi sea. ${ }^{46,75}$ We should be sufficiently careful to use GA to study a Mott transition. Nevertheless, it is fair to mention that the results of GA with respect to $\mathrm{SC}$ are often consistent with the present ones shown in $\S 5$.

\section{Appendix B: Mott transition by doublon-holon binding factors}

Up to this time, a Mott transition has been repeatedly studied using the doublon-holon binding factor $\mathcal{P}_{Q}$ and its minor variations. However, the course of study has not been straight but a little confusing. In this Appendix, we first recapitulate this subject (§B.1), and then make comments on misunderstandings based on the above confusion in a recent VMC study ${ }^{23}$ (§B.2).

\section{B.1 Mott transition by $\mathcal{P}_{Q}$}

For simplicity, let us consider the case of $t^{\prime}=0\left(\mu^{\prime}=\right.$ 0 ), which makes no substantial difference for finite $t^{\prime}$. Because $\mu$ in eq. (4) is close to 1 near a Mott transition, the wave function $\Psi_{Q}$ eq. (6) is expanded with respect to $1-\mu(=m)$ as

$$
\begin{aligned}
\Psi_{Q}=\Psi_{Q}^{(0)}+m \Psi_{Q}^{(1)}+m^{2} \Psi_{Q}^{(2)} \cdots, \\
\Psi_{Q}^{(0)}=\left[\prod_{i}\left(1-Q_{i}\right)\right] \Psi_{\mathrm{G}}, \\
\Psi_{Q}^{(1)}=\left\{\sum_{j} Q_{j}\left[\prod_{i(\neq j)}\left(1-Q_{i}\right)\right]\right\} \Psi_{\mathrm{G}}, \\
\Psi_{Q}^{(2)}=\left\{\sum_{j, k(\neq j)} Q_{j} Q_{k}\left[\prod_{i(\neq j, k)}\left(1-Q_{i}\right)\right]\right\} \Psi_{\mathrm{G}} .
\end{aligned}
$$

The bases that compose $\Psi_{Q}^{(\ell)}$ possess necessarily $\ell$ lattice sites which are occupied by doublons (or holons) without a holon (doublon) in their nearest-neighbor sites. Note that in the vicinity of a Mott transition, $g$ (Gutzwiller factor) is so small that the doublon density is very low $(D \lesssim 0.04$, as shown in Fig. 8). Using eq. (B.1), the kinetic and interaction energies are formally expanded with respect to $m$, as

$$
\begin{aligned}
E_{\text {kin }}(g, m) & =K_{0}+m K_{1}+m^{2} K_{2}+\cdots, \\
E_{\text {int }}(g, m) & =I_{0}+m^{2} I_{2}+\cdots,
\end{aligned}
$$

in which $K_{\ell}$ and $I_{\ell}(\ell=0,1,2, \cdots)$ are functions of the Gutzwiller factor $g$

$$
\begin{gathered}
K_{0}+I_{0}=E_{0}=\langle 0|\mathcal{H}| 0\rangle / N_{0} \\
K_{1}=\left[\left\langle 0\left|\mathcal{H}_{\text {kin }}\right| 1\right\rangle+\left\langle 1\left|\mathcal{H}_{\text {kin }}\right| 0\right\rangle\right] / N_{0} \\
K_{2}+I_{2}=\left[\left\langle 0\left|\mathcal{H}_{\text {kin }}\right| 2\right\rangle+\left\langle 1\left|\mathcal{H}_{\text {kin }}\right| 1\right\rangle+\left\langle 2\left|\mathcal{H}_{\text {kin }}\right| 0\right\rangle+\right. \\
\left.\left\langle 1\left|\mathcal{H}_{\text {int }}\right| 1\right\rangle+N_{1}\langle 0|\mathcal{H}| 0\rangle\right] / N_{0}
\end{gathered}
$$

with $\left|\Psi_{Q}^{(\ell)}\right\rangle \equiv|\ell\rangle$ and $N_{\ell}=\langle\ell \mid \ell\rangle$. Thus, the total energy is given as

$$
E(g, m)=E_{0}+m K_{1}+m^{2}\left(K_{2}+I_{2}\right)+\cdots .
$$

From eq. (B.10), it seems that $E(g, m)$ has a minimum at a finite $m$ (or $\mu<1$ ), if $K_{1}$ is negative. Actually, Yokoyama and Shiba ${ }^{21}$ calculated $K_{1}$ for onedimensional systems, and confirmed that $K_{1}$ is negative. They thus concluded that $m$ is always finite as long as $U / t$ is finite, that is, a Mott transition does not arise.

Later, however, this conclusion turned out to be substantially incorrect through a more careful study. ${ }^{20}$ Because, at half filling, a doublon and a holon are created simultaneously as a pair, a basis included in $\Psi_{Q}^{(1)}$ has at least two doublons, whereas $\Psi_{Q}^{(2)}$ contains bases with only one doublon, as shown in Fig. B·1. Thus, as $g$ decreases, $\left|K_{1}\right|$ can become negligible, compared with $\left|K_{2}\right|$. In fact, $K_{1} / t$ has been calculated using VMC with $\mathcal{P}_{Q} \mathcal{P}_{\mathrm{G}} \Phi_{\mathrm{F}}$ for the one-dimensional Hubbard model, with the result that as $g$ decreases, $\left|K_{1}\right| / t$ is abruptly reduced from $\sim 0.1(g=0.4)$ to $\sim 0.007(g=0.2) .{ }^{21}$ In addition, the quadratic behavior of $E(m, g)$ for small $g$ and $m \rightarrow 0$, namely $\partial E / \partial m=0$, is clearly observed for twodimensional cases. ${ }^{20}$ Eventually, it was confirmed that Mott transitions actually take place for the square lattice Hubbard model by applying $\mathcal{P}_{Q}$ to $\Phi_{\mathrm{F}}{ }^{20}$ and $\Phi_{d}{ }^{19}$ Afterward, Mott transitions have been observed using $\mathcal{P}_{Q}$ for various systems in addition to the present model: the lattice shown in Fig.1(b) with both $\Phi_{\mathrm{F}}$ and $\Phi_{d},{ }^{37}$ the kagomé lattice with $\Phi_{\mathrm{F}},{ }^{38}$ the checker-board lattice with $\Phi_{\mathrm{F}},{ }^{39}$ and a degenerate Hubbard model on the square lattice with $\Phi_{\mathrm{F}} \cdot{ }^{40}$

Independently of ref. 21, Millis and Coppersmith ${ }^{22}$ studied the possibility of a Mott transition with a virtually equivalent variational wave function to $\mathcal{P}_{Q} \mathcal{P}_{\mathrm{G}} \Phi_{\mathrm{F}}$. To determine whether or not a Mott transition arises, they adopted a general theory of $\mathrm{Kohn}^{78}$ in estimating a zero-frequency part of the optical conductivity from the linear response of twisted-boundary systems to a small flux. They concluded that a wave function like $\mathcal{P}_{Q} \mathcal{P}_{\mathrm{G}} \Phi_{\mathrm{F}}$ never becomes insulating for a finite $U / t$, on the basis of 
(a)

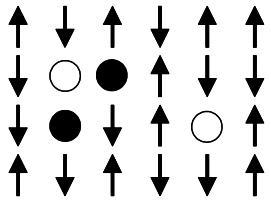

(b)

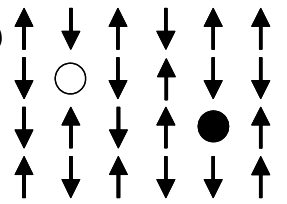

Fig. B·1. Examples of electronic configurations appearing (a) in $\Psi_{Q}^{(1)}$ and (b) in $\Psi_{Q}^{(2)}$, at half filling. Solid (open) circles indicate doublons (holons). Each example belongs to the case in which the doublon number is minimum.

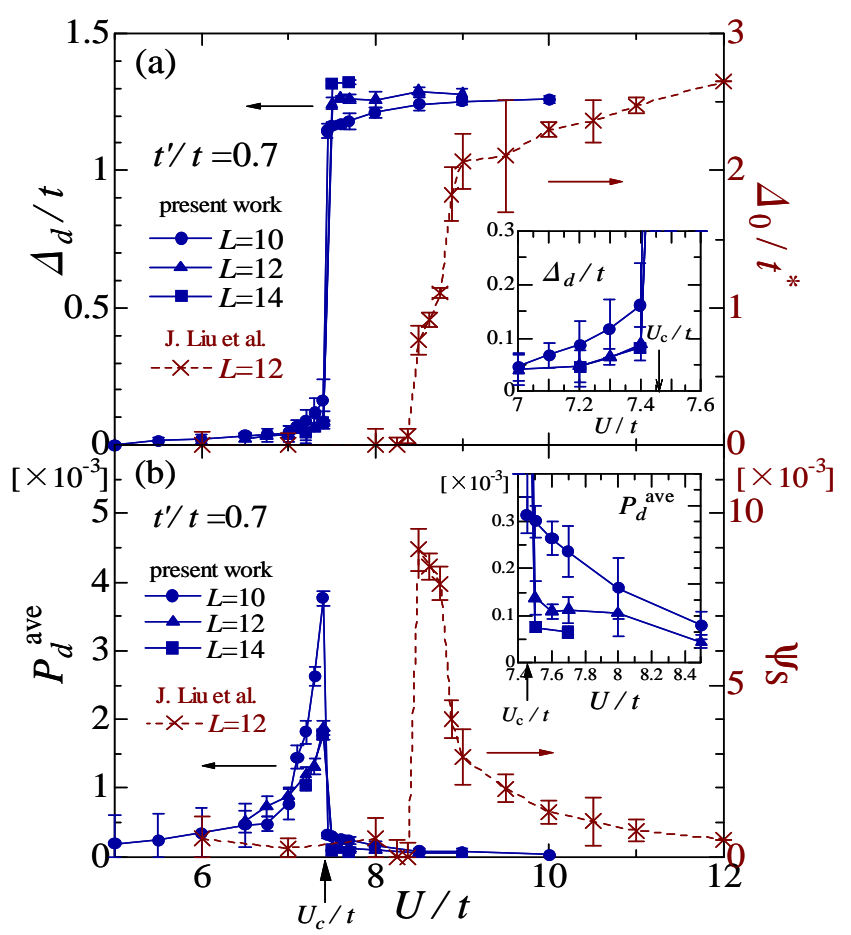

Fig. B.2. (Color online) Comparison of VMC results for $t^{\prime} / t=$ 0.7 between the study of Liu et al. (taken from Fig. 1 in ref. 23) and the present study for (a) the $d$-wave gap (the optimized variational parameters), and (b) the pairing correlation function. The quantities studied by Liu et al., $\Delta_{0} / t^{*}$ and $\psi_{\mathrm{s}}$, (crosses) practically correspond to $\Delta_{d} / t$ and $P_{d}^{\text {ave }}$ in the present study (solid symbols), respectively.

a VMC result with a nonoptimized $g(=0.3)$ for a onedimensional small (10-site) system. However, this conclusion is an error, as we have demonstrated with actual counterevidence in the preceding paragraph (or in $\S 4$ ). We need to check this study as to which process causes the error.

\section{B.2 Comments on recent VMC study}

Recently, Liu et al. ${ }^{23}$ have studied the same model eq. (1) using a VMC method with an almost identical $d$ wave singlet trial function (abbreviated as $\Psi_{\text {Liu }}$ ) to ours, $\Psi_{Q}^{d}$, in eq. (6). The differences between $\Psi_{\text {Liu }}$ and $\Psi_{Q}^{d}$ are as follows: (i) In $\Psi_{\mathrm{Liu}}$, to improve the renormalization effect of the quasi-Fermi surface, $\tilde{t}^{\prime \prime}$ is additionally introduced in the other diagonal $(-1,1)$ direction in $\tilde{\varepsilon}_{\mathbf{k}}$ as a parameter. (ii) In $\Psi_{\text {Liu }}$, the magnitude of the doublonholon binding factors for next-nearest-neighbor sites is assumed identical to that for the nearest-neighbor ones,

namely $\mu^{\prime}=\mu$ in our notation. Because these differences are not essential, ${ }^{79}$ it is natural that the results of Liu et al. are not profoundly different from the present ones, and that some of their arguments are common to ours; we are ready to respect this aspect of their study. However, in a crucial point in connection with a Mott transition, their interpretation is distinct from ours and questionable. Because it is vital to clarify the properties of Mott transitions in this type of wave function for related studies, it is necessary to clarify the questionable points.

In the study of Liu et al., a pair correlation function $\left(\psi_{s}\right)$ was calculated for $t^{\prime} / t=0.7$ with a fixed system size, $L=12$. We have traced $\psi_{s}$ from Fig. 1 in ref. 23, and plotted it in Fig. B.2(b) together with our corresponding results. For small values of $U / t, \psi_{s}$ is negligible, whereas it increases at approximately $U / t=8.5$ and then it gradually decreases. This behavior of $\psi_{s}$ is interpreted in ref. 23 as follows: For small values of $U / t$, the state is a conventional metal; robust SC occurs at approximately $U / t=8.5$, and weak $S C$ continues to larger values of $U / t$ without a transition. The problematic interpretation consists in the last point. In comparing $\psi_{\mathrm{s}}$ with $P_{d}^{\text {ave }}, U / t$ at which $\psi_{s}$ becomes conspicuously large $(\sim 8.5)$ is larger roughly by 1 than $U_{\mathrm{c}} / t(=7.45)$ of $P_{d}^{\text {ave }}$. This discrepancy may stem from point (ii) of the differences in the wave functions,${ }^{79}$ and is of little importance. The most crucial point is that they overlooked the Mott transition this type of wave functions exhibits immediately above the region of robust SC. They probably accepted the incorrect claim of Millis and Coppersmith mentioned in $\S$ B.1, and neglected to check the system-size dependence of $\psi_{\mathrm{s}}{ }^{80}$ For such a finite system as $L=12, \psi_{\mathrm{s}}$ is still visibly finite, but must decrease abruptly with increasing $L$, as we showed the system-size dependence of $P_{d}^{\text {ave }}$ in the inset of Fig. B·2(b).

This misinterpretation influences some other points. Because the region of SC is considered to continue to larger values of $U / t$ ( $\gtrsim 8.7$ ), the features of the insulating state are confused with those of the SC state. Although the prominent renormalization of $\varepsilon_{\mathbf{k}}$ (or $t^{\prime}$ and $t^{\prime \prime}$ ) is considered to occurs in the SC state, as shown in Fig. 3 of ref. 23; correctly, the renormalization of $t^{\prime} / t$ is small in the SC state, but prominent in the insulating state, as we showed in $\S 4$ and $\S 5$. Furthermore, the spin correlation function is compared between the metallic regime $(U / t=7)$, for which $\psi_{\mathrm{s}}$ is negligibly small, and the insulating regime $(U / t=11)$ in Fig. 4 of ref. 23. Therefore, as long as the spin correlation in the SC state is concerned, this comparison is useless.

Finally, we point out the possible fictitious enhancement of $P_{d}^{\text {ave }}\left(\right.$ or $\psi_{\mathrm{s}}$ ) specific to finite system sizes. In Fig. B.2(b), the values of $P_{d}^{\text {ave }}$ immediately below $U_{\mathrm{c}}$ for $L=10$ are roughly twice larger than those for $L=12$ and 14. This is because the $k$-point configuration for $L=10$ happens to be specially advantageous for SC under the given condition, including the renormalized effect of $\varepsilon_{\mathbf{k}}$ by $U$. This is confirmed by the fact that the quasiFermi surface has a different shape only in these special cases (not shown). Undoubtedly, the behavior of $P_{d}^{\text {ave }}$ for $L=12$ and 14 will be closer to that for $L=\infty$. In accor- 
dance with this anomalous enhancement in $P_{d}^{\text {ave }}, \Delta_{d} / t$ takes large values as observed in the inset of Fig. B·2(a). According to our experience, ${ }^{37}$ such behavior is not rare for the marginal values of $t^{\prime} / t$ where $\mathrm{SC}$ is vanishing. When we return to the data of $\Psi_{\text {Liu }}$ in Fig. B·2(a) with this situation in mind, we notice that the values of $\Delta_{0} / t^{*}$ for $U / t \sim 8.5$ (three points), where $\psi_{\mathrm{s}}$ is considerably enhanced, are specifically increased. Hence, the prominent peak in $\psi_{\mathrm{s}}$ at $U / t \sim 8.5$ is probably a fictitious increase specific to their system size and boundary conditions.

We hope improved studies of $\Psi_{\text {Liu }}$ will appear.

1) K. Kanoda: Physica C 282-287 (1997) 299; K. Miyagawa, K. Kanoda and A. Kawamoto: Chem. Rev. 104 (2004) 5635.

2) R. H. McKenzie: Science 278 (1997) 820.

3) R. H. McKenzie: Comments Cond. Mat. Phys. 18 (1998) 309.

4) Y. Shimizu, K. Miyagawa, K. Kanoda, M. Maesato and G. Saito: Phys. Rev. Lett. 91 (2003) 107001.

5) H. Kino and H. Fukuyama: J. Phys. Soc. Jpn. 65 (1996) 2158.

6) H. Kino and H. Kontani: J. Phys. Soc. Jpn. 67 (1998) 3691.

7) H. Kondo and T. Moriya: J. Phys. Soc. Jpn. 67 (1998) 3695.

8) K. Kuroki and H. Aoki: Phys. Rev. B 60 (1999) 3060

9) H. Mayaffre, P. Wzietec, D. Jerome, C. Lenoir and P. Batail: Phys. Rev. Lett. 75 (1995) 4122.

10) S. M. De Soto, C. P. Slichter, A. M. Kini, H. H. Wang, U. Geiser and J. M. Williams: Phys. Rev. B 52 (1995) 10364.

11) K. Kanoda, K. Miyagawa, A. Kawamoto and Y. Nakazawa: Phys. Rev. B 54 (1996) 76.

12) K. Izawa, H. Yamaguchi, T. Sasaki and Y. Matsuda: Phys. Rev. Lett. 88 (2001) 027002.

13) T. Arai, K. Ichimura, K. Nomura, S. Takasaki, J. Yamada, S. Nakatsuji and H. Anzai: Phys. Rev. B 63 (2001) 104518.

14) K. Ichimura, S. Higashi, K. Nomura and A. Kawamoto: Synthetic Metals 153409 (2005).

15) Y. Tanuma, K. Kuroki, Y. Tanaka and S. Kashiwaya: Phys. Rev. B 68 (2003) 214513.

16) In this paper, we use the term 'Mott transition' for a transition from a conductive state to a nonmagnetic insulating state. Thus, we discriminate it from a magnetic transition like a metal-to-AF-insulator transition.

17) O. Parcollet, G. Biroli and G. Kotliar: Phys. Rev. Lett. 92 (2004) 226402.

18) H. Morita, S. Watanabe and M. Imada: J. Phys. Soc. Jpn. 71 (2002) 2109.

19) H. Yokoyama, Y. Tanaka, M. Ogata and H. Tsuchiura: J. Phys. Soc. Jpn. 73 (2004) 1119.

20) H. Yokoyama: Prog. Theor. Phys. 108 (2002) 59.

21) H. Yokoyama and H. Shiba: J. Phys. Soc. Jpn. 59 (1990) 3669.

22) A. J. Millis and S. N. Coppersmith: Phys. Rev. B 43 (1991) 13770.

23) J. Liu, J. Schmalian and N. Trivedi: Phys. Rev. Lett. 94 (2005) 127003.

24) T. Watanabe, H. Yokoyama, Y. Tanaka and J. Inoue: to appear in Physica C.

25) T. Komatsu, N. Matsukawa, T. Inoue and G. Saito: J. Phys. Soc. Jpn. 65 (1996) 1340.

26) M. Tamura, H. Tajima, K. Yakushi, H. Kuroda, A. Kobayashi, R. Kato and H. Kobayashi: J. Phys. Soc. Jpn. 60 (1991) 3861.

27) At half filling, the results for $t^{\prime}(<0)$ are identical to those for $\left|t^{\prime}\right|$. We hence concentrate on the case of $t^{\prime} / t \geq 0$.

28) C. J. Umrigar, K. G. Wilson and J. W. Wilkins: Phys. Rev. Lett. 60 (1988) 1719.

29) M. C. Gutzwiller: Phys. Rev. Lett. 10 (1963) 159

30) H. Yokoyama and M. Ogata: J. Phys. Soc. Jpn. 65 (1996) 3615.

31) A. Paramekanti, M. Randeria and N. Trivedi: Phys. Rev. B 70 (2004) 054504

32) H. Yokoyama and H. Shiba: J. Phys. Soc. Jpn. 56 (1987) 1490.

33) M. Capello, F. Becca, S. Yunoki and S. Sorella: cond- mat/0509062.

34) T. A. Kaplan, P. Horsch and P. Fulde: Phys. Rev. Lett. 49 (1982) 889.

35) Because the $t-J$ and Hubbard models are related in the strong coupling regime through a canonical transformation, $\mathcal{H}_{t-J} \approx$ $e^{i S} \mathcal{H}_{\mathrm{Hub}} e^{-i S}, 36$ in which $S$ is given as

$$
\sum_{\langle i j\rangle \sigma} \frac{-i t}{U}\left[n_{i-\sigma}\left(1-n_{j-\sigma}\right) c_{i \sigma}^{\dagger} c_{j \sigma}-n_{j-\sigma}\left(1-n_{i-\sigma}\right) c_{i \sigma}^{\dagger} c_{j \sigma}\right],
$$

the expectation values of the $t-J$ model with respect to $\Psi_{\mathrm{G}}$ are approximately equivalent to those of the Hubbard model with respect to $e^{-i S} \Psi_{\mathrm{G}}$. When one expands $e^{-i S}$, the firstorder part $(\sim t / U)$ of $e^{-i S} \Psi_{\mathrm{G}}$ broadly corresponds to $\mathcal{P}_{\mathrm{Q}} \Psi_{\mathrm{G}}$. Thus, the favorable feature in treating the $t-J$ model with $\Psi_{\mathrm{G}}$ is shared with the case in which the Hubbard model is treated with $\mathcal{P}_{\mathrm{Q}} \Psi_{\mathrm{G}}$.

36) A. B. Harris and R. V. Lange: Phys. Rev. 157 (1967) 295.

37) H. Yokoyama, Y. Tanaka and M. Ogata: in preparation.

38) S. Kuratani, A. Koga and N. Kawakami: private communication.

39) A. Koga, T. Yoshioka, N. Kawakami: private communication.

40) A. Koga, N. Kawakami, H. Yokoyama and K. Kobayashi: proceedings of LT24.

41) A. Himeda and M. Ogata: Phys. Rev. Lett. 85 (2000) 4345.

42) It is difficult to estimate proper values of $Z$ as a function of $U / t$ with a finite-sized system, in the case that the position of the quasi-Fermi point moves and crosses a $k$ point, when $U / t$ is varied. As an example, we show the case of $t^{\prime} / t=0.4$ and $L=12$ in Fig. 6, where the data for $U / t=6.5$ deviates to some extent. For this parameter set, we find the quasi-Fermi point to shift in the nodal direction in Fig. 5(a).

43) R. Bulla: Phys. Rev. Lett. 83 (1999) 136.

44) M. J. Rozenberg, R. Chitra and G. Kotliar: Phys. Rev. Lett. 83 (1999) 3498.

45) Generally, as system size decreases, the critical behavior near a phase transition tends to be less sharp, because a transition, in a strict sense, takes place only for $N_{\mathrm{s}} \rightarrow \infty$. For instance, the curve for $t^{\prime}=0$ and $L=10$ in Fig. 8 (open square) is relatively smooth, whereas the curve for $L=16$ seems to have an obvious jump. Thus, one must necessarily check system-size dependence to treat a phase transition.

46) W. F. Brinkman and T. M. Rice: Phys. Rev. B 2 (1970) 4302.

47) W. Metzner and D. Vollhardt: Phys. Rev. B 37 (1988) 7382.

48) P. G. J. van Dongen, F. Gebhard and D. Vollhardt: Z. Phys. B 76 (1989) 199; F. Gebhard: Phys. Rev. B 41 (1990) 9452.

49) M. C. Gutzwiller: Phys. Rev. 137 (1965) A1726; D. Vollhardt: Rev. Mod. Phys. 56 (1984) 99.

50) J. Y. Gan, Y. Chen, Z. B. Su and F. C. Zhang: Phys. Rev. Lett. 94 (2005) 067005.

51) B. J. Powell and R. H. McKenzie: Phys. Rev. Lett. 94 (2005) 047004.

52) F. C. Zhang, C. Gros, T. M. Rice and H. Shiba: Supercond. Sci. Tech. 1 (1988) 36.

53) For instance, A. Georges, G. Kotliar, W. Krauth and M. J. Rozenberg: Rev. Mod. Phys. 68 (1996) 13.

54) For instance, M. Imada: Phys. Rev. B 72 (2005) 075113.

55) We adopt the Manhattan metric to measure $|\mathbf{R}|$ and $|\mathbf{r}|$.

56) When $U / t$ is small and the coherence length is large, one needs to consider SC correlation functions of longer Cooper pairs. In the present study, however, $P_{d}(\mathbf{r})$ in eq. (17) is useful, because we are interested in the SC arising, particularly, near the Mott transition.

57) The weak correlation regimes, we here assume, are $U / t<6$ for $t^{\prime} / t=0.2,0.4$, and 0.6 , and $U<U_{\mathrm{c}}$ for $t^{\prime} / t=0.7$ and 0.8 .

58) K. Tanaka, H. Ikeda and K. Yamada: J. Phys. Soc. Jpn. 73 (2004) 3394.

59) T. Watanabe, H. Yokoyama, Y. Tanaka, J. Inoue and M. Ogata: Physica C 426-431 (2005) 289.

60) W. Zheng, R. R. P. Singh, R. H. McKenzie and R. Coldea: Phys. Rev. B 71 (2005) 134422.

61) Y. Kurosaki, Y. Shimizu, K. Miyagawa, K. Kanoda and G. Saito: Phys. Rev. Lett. 95 (2005) 177001. 
62) This feature is similar to that in the inorganic spin-Peierls compound $\mathrm{CuGeO}_{3}$, in which the value of $J^{\prime} / J$ drastically varies (including sign) under pressure. See e.g., H. Yokoyama and Y. Saiga: J. Phys. Soc. Jpn. 66 (1997) 3617; S. Watanabe and H. Yokoyama: J. Phys. Soc. Jpn. 68 (1999) 2073.

63) M. Rahal, D. Chasseau, J. Gaultier, L. Ducasse, M. Kurmoo and P. Day: Acta. Cryst. B 53 (1997) 159.

64) C. E. Campos, P. S. Sandhu, J. S. Brooks and T. Ziman: Phys. Rev. B 53 (1996) 12725.

65) C. Weber, A. Laeuchli, F. Mila and T. Giamarchi: Phys. Rev. B 73 (2006) 014519; O. I. Motrunich: Phys. Rev. B 72 (2005) 045105.

66) P. W. Anderson: Science 235 (1987) 1196.

67) For instance, W. Zheng, R. H. McKenzie and R. R. P. Singh: Phys. Rev. B 59 (1999) 14367; L. O. Manuel and H. A. Ceccatto: Phys. Rev. B 60 (1999) 9489.

68) T. Giamarchi and C. Lhuillier: Phys. Rev. B 43 (1991) 12943; A. Himeda and M. Ogata: Phys. Rev. B 60 (1999) 9935.

69) S. Liang, B. Doucot and P. W. Anderson: Phys. Rev. Lett. 61 (1988) 365

70) H. Yokoyama and H. Shiba: J. Phys. Soc. Jpn. 57 (1988) 2482; C. Gros: Phys. Rev. B 38 (1988) 931.

71) T. Watanabe, H. Yokoyama, Y. Tanaka, J. Inoue and M Ogata: J. Phys. Soc. Jpn. 73 (2004) 3404.

72) H. Kondo and T. Moriya: J. Phys. Soc. Jpn. 73 (2004) 812.

73) J. Schmalian: Phys. Rev. Lett. 81 (1998) 4232; K. Kuroki,
T. Kimura, R. Arita, Y. Tanaka and Y. Matsuda: Phys. Rev. B 65 (2002) 100516.

74) S. Kashiwaya and Y. Tanaka: Rep. Prog. Phys. 63 (2000) 1641; Y. Tanaka and S. Kashiwaya: Phys. Rev. Lett. 74 (1995) 3451.

75) T. Ogawa, K. Kanda and T. Matsubara: Prog. Theor. Phys. 53 (1975) 614.

76) H. Kondo and T. Moriya: J. Phys. Soc. Jpn. 65 (1995) 2559.

77) H. Yokoyama and H. Shiba: J. Phys. Soc. Jpn. 56 (1987) 3582.

78) W. Kohn: Phys. Rev. 133 (1964) A171.

79) The effect of difference (i), namely of $\tilde{t}^{\prime \prime} / t$, must be slight, because, according to Fig. 2 in ref. 23, the optimized $\left|\tilde{t}^{\prime \prime} / t\right|$ is small both for the conductive $(U / t \lesssim 8.5)$ and insulating $(U / t \gtrsim 8.5)$ phases. The effect of difference (ii), namely of putting $\mu^{\prime}=\mu$ in $\Psi_{\text {Liu }}$, manifests itself to some extent, because, as in Figs. 4(d) and 4(e), the optimized values of $\mu$ and $\mu^{\prime}$ are quite different, especially in the insulating phase. Thus, it is possible that $\Psi_{\text {Liu }}$ becomes quantitatively worse than $\Psi_{Q}^{d}$ for $U \gtrsim U_{\mathrm{c}}$; better results would be obtained, if $\mu^{\prime}=0$ is used instead of $\mu^{\prime}=\mu$ in $\Psi_{\text {Liu }}$.

80) In ref. 23, the system-size dependence of the gap parameter $\Delta_{0} / t^{*}$ for $U / t=9.5$ (in the insulating regime) is shown in the inset of Fig. 1. Because the finite $\Delta_{0} / t^{*}$ does not necessarily indicate the realization of SC, as we have mentioned, it is useless to check whether $\Delta_{0} / t^{*}$ remains finite for $L \rightarrow \infty$, in order to determine whether or not SC arises. 